\title{
Cx43 hemichannel microdomain signaling at the intercalated disc enhances cardiac excitability
}

\author{
Maarten A.J. De Smet, ${ }^{1,2,3}$ Alessio Lissoni, ${ }^{1}$ Timur Nezlobinsky, ${ }^{4,5}$ Nan Wang, ${ }^{1}$ Eef Dries, ${ }^{2}$ Marta Pérez-Hernández, ${ }^{6}$ Xianming Lin, ${ }^{6}$ \\ Matthew Amoni, ${ }^{2}$ Tim Vervliet, ${ }^{7}$ Katja Witschas, ${ }^{1}$ Eli Rothenberg, ${ }^{8}$ Geert Bultynck, ${ }^{7}$ Rainer Schulz, ${ }^{9}$ Alexander V. Panfilov, ${ }^{4,5,10}$ \\ Mario Delmar, ${ }^{6}$ Karin R. Sipido, ${ }^{2}$ and Luc Leybaert ${ }^{1}$ \\ ${ }^{1}$ Physiology Group, Department of Basic and Applied Medical Sciences, Chent University, Chent, Belgium. ${ }^{2}$ Experimental Cardiology, Department of Cardiovascular Sciences, KU Leuven, Leuven, Belgium. \\ ${ }^{3}$ Department of Internal Medicine and ${ }^{4}$ Department of Physics and Astronomy, Ghent University, Ghent, Belgium. ${ }^{5}$ Laboratory of Computational Biology and Medicine, Ural Federal University, Ekaterinburg, \\ Russia. ${ }^{6}$ Leon H. Charney Division of Cardiology, School of Medicine, New York University, New York, New York, USA. 'Laboratory of Molecular and Cellular Signaling, Department of Cellular and Molecular \\ Medicine, KU Leuven, Leuven, Belgium. ${ }^{8}$ Department of Biochemistry and Molecular Pharmacology, School of Medicine, New York University, New York, New York, USA. ${ }^{9}$ Physiologisches Institut, \\ Justus-Liebig-Universität, Giessen, Germany. ${ }^{10}$ Arrhythmia Department, Almazov National Medical Research Centre, Saint Petersburg, Russia.
}

Cx43, a major cardiac connexin, forms precursor hemichannels that accrue at the intercalated disc to assemble as gap junctions. While gap junctions are crucial for electrical conduction in the heart, little is known about the potential roles of hemichannels. Recent evidence suggests that inhibiting Cx43 hemichannel opening with Gap19 has antiarrhythmic effects. Here, we used multiple electrophysiology, imaging, and super-resolution techniques to understand and define the conditions underlying Cx43 hemichannel activation in ventricular cardiomyocytes, their contribution to diastolic $\mathrm{Ca}^{2+}$ release from the sarcoplasmic reticulum, and their impact on electrical stability. We showed that Cx43 hemichannels were activated during diastolic $\mathrm{Ca}^{2+}$ release in single ventricular cardiomyocytes and cardiomyocyte cell pairs from mice and pigs. This activation involved $\mathrm{Cx} 43$ hemichannel $\mathrm{Ca}^{2+}$ entry and coupling to $\mathrm{Ca}^{2+}$ release microdomains at the intercalated disc, resulting in enhanced $\mathrm{Ca}^{2+}$ dynamics. Hemichannel opening furthermore contributed to delayed afterdepolarizations and triggered action potentials. In single cardiomyocytes, cardiomyocyte cell pairs, and arterially perfused tissue wedges from failing human hearts, increased hemichannel activity contributed to electrical instability compared with nonfailing rejected donor hearts. We conclude that microdomain coupling between Cx43 hemichannels and $\mathrm{Ca}^{2+}$ release is a potentially novel, targetable mechanism of cardiac arrhythmogenesis in heart failure.

\section{Introduction}

The pumping function of the heart is coordinated by electrical waves of excitation propagating through the myocardium and initiating cardiac contraction. Propagation of action potentials between cardiomyocytes is coordinated by gap junctions that accrete at the intercalated discs. Each gap junction channel consists of 2 apposed hemichannels and each hemichannel is further composed of 6 connexin (Cx) subunits; $\mathrm{Cx} 43$ is the predominant $\mathrm{Cx}$ isotype in adult heart ventricles (1). Gap junctions organize as channel plaques, called the nexus, at the intercalated discs of adjacent cardiomyocytes, which are peripherally surrounded by perinexal zones where unapposed (free) hemichannels reside (2); at rest, gap junctions are open and hemichannels are closed. In ventricular cardiomyocytes, $\mathrm{Cx} 43$ hemichannels need strongly positive membrane voltages $\left(\mathrm{V}_{\mathrm{m}}>+50 \mathrm{mV}\right)$ to open; the activation threshold is lowered when the cytoplasmic $\mathrm{Ca}^{2+}$ concen-

Authorship note: MD, KRS, and LL are co-senior authors. Conflict of interest: The authors have declared that no conflict of interest exists. Copyright: () 2021, American Society for Clinical Investigation.

Submitted: March 16, 2020; Accepted: February 19, 2021; Published: April 1, 2021 Reference information: J Clin Invest. 2021;131(7):e137752.

https://doi.org/10.1172/JCl137752. tration $\left(\left[\mathrm{Ca}^{2+}\right]_{\mathrm{i}}\right)$ is moderately increased (200-500 nM range), bringing the threshold into the $+30 \mathrm{mV}$ range, which may be attained during the peak and plateau phase of the cardiac action potential $(3,4)$. When open, hemichannels form a large conductance ( $220 \mathrm{pS}$ for a single $\mathrm{Cx} 43$ hemichannel) and a poorly selective ion channel and transmembrane conduit, with a molecular weight cutoff of approximately $1.5 \mathrm{kDa}(3-9)$ that allows the passage of various small molecules such as ATP and ions such as $\mathrm{Na}^{+}, \mathrm{K}^{+}$, and $\mathrm{Ca}^{2+}$.

Inspired by findings in noncardiac cells where $\mathrm{Cx} 43$ hemichannels open in response to moderate $\left(\mathrm{Ca}^{2+}\right)_{\mathrm{i}}$ elevation at resting $\mathrm{V}_{\mathrm{m}}(7,9)$, we were interested in whether $\mathrm{Cx} 43$ hemichannels in cardiomyocytes could open during sarcoplasmic reticulum (SR) $\mathrm{Ca}^{2+}$ release under conditions of $\mathrm{Ca}^{2+}$ overload and disease. Cardiomyocytes display synchronized transient $\left[\mathrm{Ca}^{2+}\right]_{\mathrm{i}}$ elevations during systole, sparked by $\mathrm{Ca}^{2+}$ entry through L-type $\mathrm{Ca}^{2+}$ channels during the action potential plateau, which triggers $\mathrm{Ca}^{2+}$-induced $\mathrm{SR} \mathrm{Ca}{ }^{2+}$ release via ryanodine receptors (RyRs, ref. 10). Under pathological conditions, spontaneous SR $\mathrm{Ca}^{2+}$ release may occur, leading to delayed afterdepolarizations (DADs) during diastole as a result of $\mathrm{Ca}^{2+}$ extrusion via the electrogenic $\mathrm{Na}^{+} / \mathrm{Ca}^{2+}$ exchanger (NCX) that generates depolarizing inward current $(11,12)$. DADs in turn may lead to disturbances of heart rhythm $(12,13)$. We hypothesized that 
A

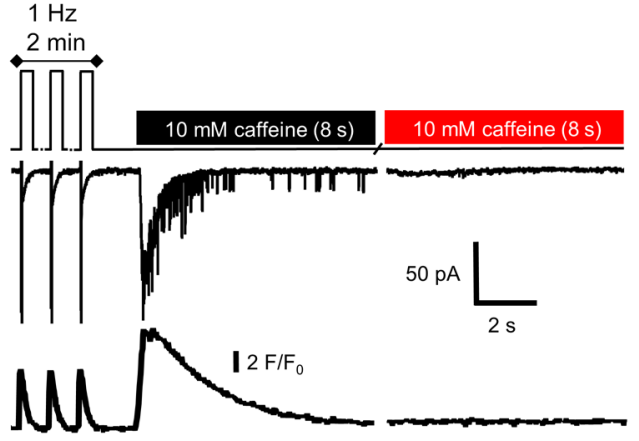

D

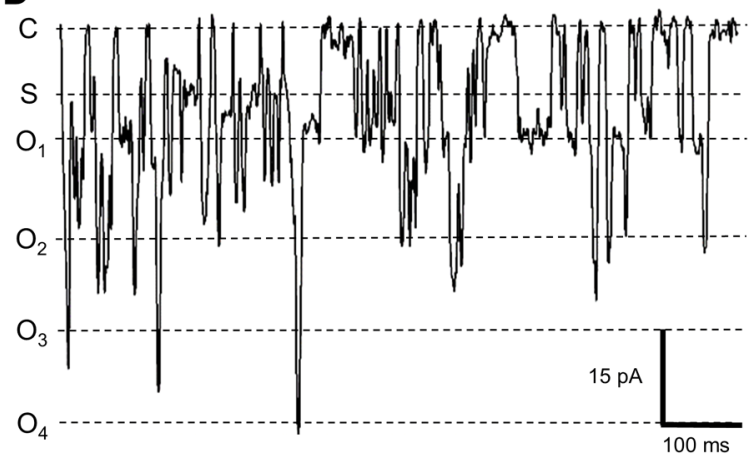

$\mathbf{F}$

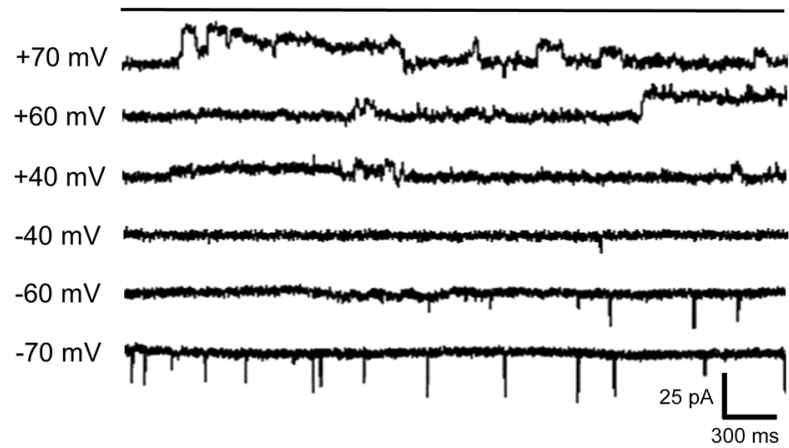

H

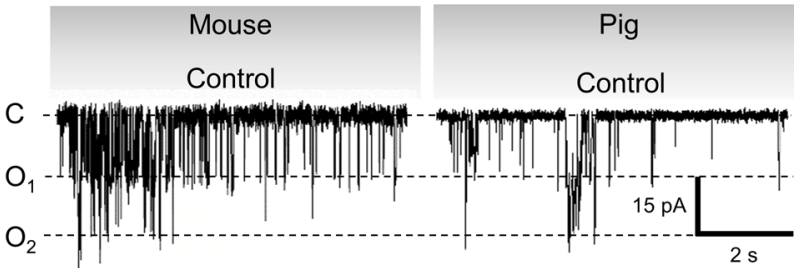

Cx43 $\mathrm{Cre-ER(T)/fl}$

$\operatorname{siC} \times 43^{1}$

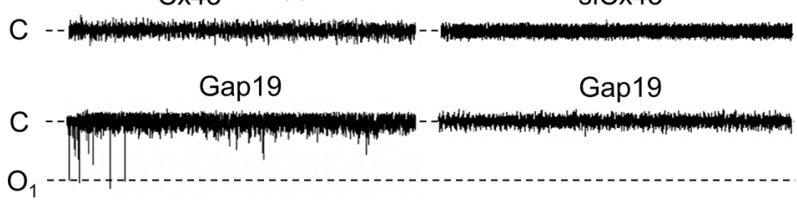

$\begin{array}{ll}\text { CT9 CT9 } & \text { CT9 }\end{array}$

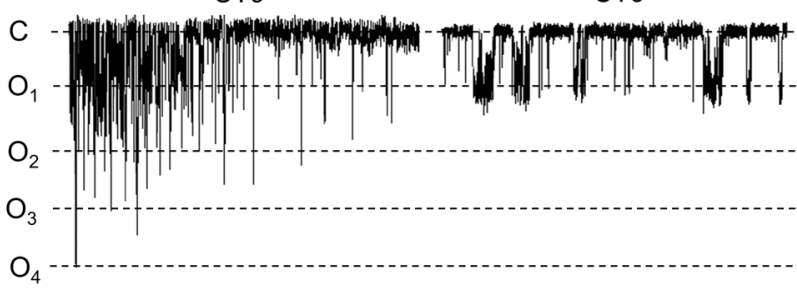

B

E
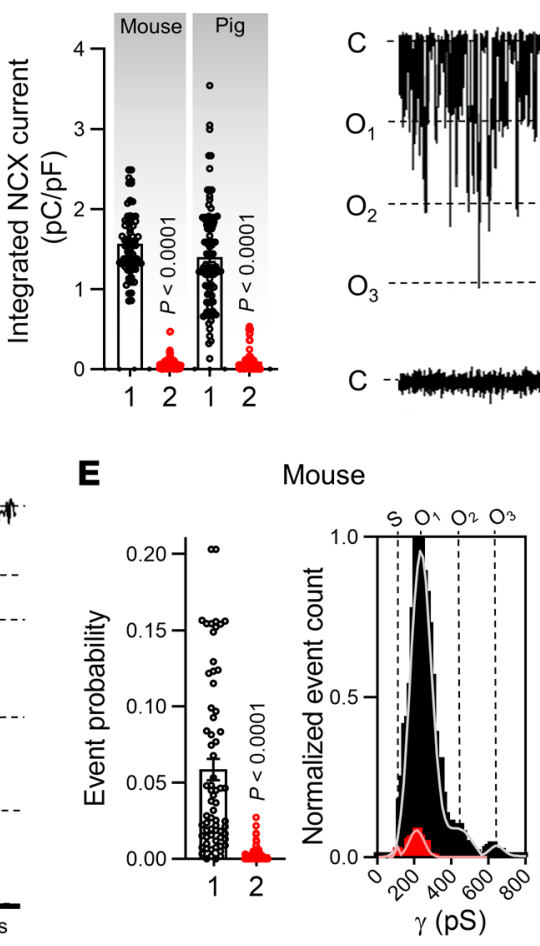

G
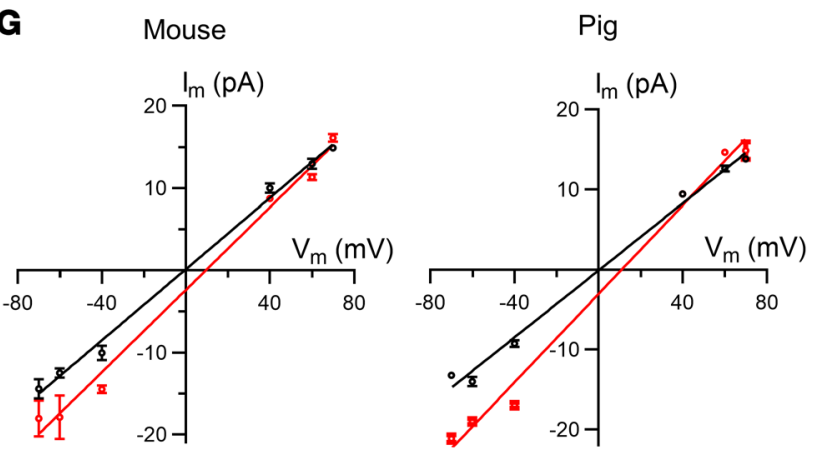

I

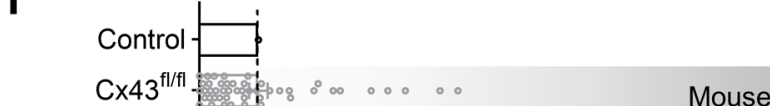

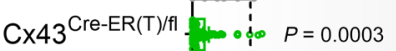

Gap19 Enow do $\quad P=0.0029$

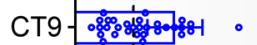

Gap $19^{1130 A}$ A

${ }^{10} \mathrm{Panx} 1$ \%

MOCK

$\mathrm{SiC} \times 43^{\mathrm{scr}}$

$\mathrm{SiC} \times 43^{1}$.

$\mathrm{siC} \times 43^{2}, \quad \circ P=0.0019$

Gap19 $\circ: \circ<0.0001$

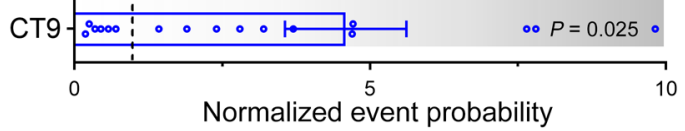


Figure 1. Caffeine-induced $\mathrm{Ca}^{2+}$ release from the sarcoplasmic reticulum activates $\mathrm{Cx} 43$ hemichannels at resting membrane potential. (A) Freshly isolated left ventricular cardiomyocytes were studied under voltage clamp with continuous $\left[\mathrm{Ca}^{2+}\right]_{i}$ recording. Top trace shows experimental protocol. Middle and bottom traces depict current and $\left[\mathrm{Ca}^{2+}\right]_{i}$ signals recorded in a mouse myocyte. (B) Summary data illustrating abolished NCX current during second caffeine pulse compared with first pulse (nested $t$ test), indicating depleted SR $\mathrm{Ca}^{2+}$ stores $\left(N / n_{\text {mouse }}=90 / 281, N / n_{\text {pig }}=20 / 55\right)$. (C) Unitary current example traces during first and second caffeine applications with NCX current subtracted. " $C$ " indicates closed state; $\mathrm{O}_{1}$ corresponds to fully open state; $\mathrm{O}_{2}$ and $\mathrm{O}_{3}$ indicate multiples of fully open state. (D) Expanded trace of unitary current activity. " $\mathrm{S}$ " indicates substate. (E) Summary dot plots and transition histograms indicate significantly reduced unitary current event probability during the second caffeine pulse (red) compared with the first application (black) (nested $t$ test; $N / n_{\text {mouse }}$ $=90 / 281, N / n_{\text {pig }}=20 / 55$ ). (F) Unitary current example traces during caffeine application at different membrane voltages. Recordings under conditions of $\mathrm{K}^{+}$channel blockade after 30 seconds of $1 \mathrm{~Hz}$ pacing. (G) IV plots depicting linear current-voltage relationship with slope conductance approximately $220 \mathrm{pS}$ and $\mathrm{E}_{\text {rev }} \approx 0 \mathrm{mV}$ (black line; $N / n_{\text {mouse }}=5 / 20, N / n_{\text {pig }}=$ 5/15). A 5 -fold elevation of $\left[\mathrm{Ca}^{2+}\right]_{\mathrm{e}}$ shifted $\mathrm{E}_{\mathrm{rev}}$ from 0 to approximately 9.5 $\mathrm{mV}$ (red line; $N / n_{\text {mouse }}=5 / 20, N / n_{\text {pig }}=5 / 15$ ). (H) Unitary current example traces under control conditions and after $[\times 43$ knockdown or application of Gap19 or CT9. (I) Summary data of $\mathrm{Ca}^{2+}$ release-induced unitary current event probability under conditions of $[x 43$ knockdown or in the presence of Gap19, inactive Gap191130A , CT9, or ${ }^{10} \mathrm{Panx} \times 1\left(N / n_{\text {mouse }}=5-16 / 20-49\right.$ per condition, $N / n_{\text {pig }}=5-6 / 15-21$ per condition). $P$ values indicate significance compared with control (nested 1-way ANOVA).

hemichannel-associated $\mathrm{Ca}^{2+}$ entry as well as hemichannel depolarizing current could enhance DADs. Recent work based on pharmacological inhibition of Cx43 hemichannel opening with Gap19, which renders hemichannels unavailable for opening without inhibiting gap junctions $(4,14)$, has demonstrated antiarrhythmic effects in muscular dystrophy in mice $(15,16)$, prevention of atrial fibrillation in myosin light chain 4-related atrial cardiomyopathy (17), and protective effects on disturbed intracellular $\mathrm{Ca}^{2+}$ homeostasis in plakophilin-2-deficient mice, a model of arrhythmogenic right ventricular cardiomyopathy (18).

We mimicked pathological spontaneous $\mathrm{Ca}^{2+}$ release by challenging freshly isolated single ventricular cardiomyocytes and cardiomyocyte cell pairs from mice and pigs with caffeine under controlled conditions. Activation of $\mathrm{Cx} 43$ hemichannels resulted from microdomain coupling of $\mathrm{Cx} 43$ hemichannels to RyRs and $\mathrm{SR} \mathrm{Ca}^{2+}$ release. We further found that $\mathrm{Cx} 43$ hemichannels contribute to spontaneous $\mathrm{Ca}^{2+}$ release events accompanying afterdepolarizations during adrenergic stimulation. In a next translational step, we studied single human ventricular cardiomyocytes and cardiomyocyte cell pairs obtained from heart failure patients, and found that $\mathrm{Cx} 43$ hemichannel opening, spontaneous $\mathrm{Ca}^{2+}$ release, and afterdepolarizations were increased compared with cardiomyocytes from nonfailing donor hearts. In arterially perfused tissue wedges, adrenergic stimulation increased the occurrence of DADs and triggered action potentials, which was clearly more pronounced in wedges from heart failure patients than from nonfailing control hearts and was suppressed by TAT-Gap19 hemichannel inhibition. Collectively, these data showed that $\mathrm{Cx} 43$ hemichannels became more active in end-stage heart failure and contributed to arrhythmogenic-triggered activities in disease, which could be suppressed by TAT-Gap19.

\section{Results}

$\mathrm{Ca}^{2+}$ release from the $S R$ at resting membrane potentials activates Cx43 hemichannels. Freshly isolated left ventricular cardiomyocytes from mouse and pig hearts were voltage clamped at -70 $\mathrm{mV}$, under physiological extracellular $\mathrm{Ca}^{2+}$ concentrations and continuous $\left[\mathrm{Ca}^{2+}\right]_{\mathrm{i}}$ monitoring with fluo-4 $(50 \mu \mathrm{mol} / \mathrm{L})$, and subjected to a 2-pulse caffeine stimulation protocol (Figure 1A). Caffeine stimulation $(10 \mathrm{mmol} / \mathrm{L})$ created controlled conditions of $\mathrm{SR} \mathrm{Ca}^{2+}$ release, mimicking the spontaneous release observed in pathological conditions. Caffeine activates RyRs (19) and triggers transient $\mathrm{SR} \mathrm{Ca}^{2+}$ release, with resulting forward-mode inward $\operatorname{NCX}$ current $(10,20)$. In line with this, the first caffeine pulse triggered a $\left[\mathrm{Ca}^{2+}\right]_{\mathrm{i}}$ transient and resulting NCX current; by contrast, the second caffeine pulse was not accompanied by $\left[\mathrm{Ca}^{2+}\right]_{i}$ elevation and NCX current, indicating $\mathrm{Ca}^{2+}$ store depletion (Figure 1B). Superimposed on the macroscopic NCX current during the first caffeine pulse, microscopic unitary current events appeared in a $\mathrm{Ca}^{2+}$ release-dependent manner (Supplemental Figure 2B; supplemental material available online with this article; https://doi. org/10.1172/JCI137752DS1). Such events were absent during the second caffeine pulse and were abolished by including the $\mathrm{Ca}^{2+}$ chelator BAPTA $(10 \mathrm{mmol} / \mathrm{L})$ in the pipette (Figure $1, \mathrm{C}-\mathrm{E}$ and Supplemental Figure 3). Additionally, imposing stably buffered $\left[\mathrm{Ca}^{2+}\right]_{\mathrm{i}}$ did not induce unitary currents, indicating that unitary current activity was dependent on $\mathrm{SR} \mathrm{Ca}^{2+}$ release rather than on caffeine or $\left[\mathrm{Ca}^{2+}\right]_{i}$ elevation itself (Supplemental Figure 4) as previously noted (21). Since $\mathrm{Ca}^{2+}$ release in cardiomyocytes is not homogeneous but characterized by differences in bulk cytoplasmic and subsarcolemmal $\left[\mathrm{Ca}^{2+}\right]_{\mathrm{i}}(22)$, we probed the involvement of $\left[\mathrm{Ca}^{2+}\right]_{\mathrm{i}}$ microdomains in unitary current activation by plotting ensemble current versus subsarcolemmal $\left[\mathrm{Ca}^{2+}\right]_{\mathrm{i}}$ as derived from the NCX current (ref. 23 and Supplemental Figure 2C). Ensemble currents showed no hysteresis in a low $\left[\mathrm{Ca}^{2+}\right]_{\mathrm{i}}$ range of NCXderived subsarcolemmal measurements, but hysteresis became apparent at higher concentrations resulting from hemichannel closure at high $\left[\mathrm{Ca}^{2+}\right]_{\mathrm{i}}(21)$.

Unitary events in response to $\mathrm{SR} \mathrm{Ca}^{2+}$ release could be readily observed in approximately $96 \%$ of mouse myocytes and approximately $60 \%$ of pig myocytes, with a representative event count of approximately 38 and approximately 6, respectively, over the 8 -second recording window (Supplemental Table 6). Most unitary current activity occurred within less than $100 \mathrm{~ms}$ after the start of the NCX current and had an approximately $220 \mathrm{pS}$ unitary conductance in both species (Figure 1, C-E), typical for $\mathrm{Cx} 43$ hemichannel opening $(3,4,6)$. An approximately $110 \mathrm{pS}$ substate could also be resolved, especially in the pig myocytes. Occasionally, we observed "stacked" opening events that had a conductance of multiples of approximately $220 \mathrm{pS}$ (Figure 1, C-E). Stepping $\mathrm{V}_{\mathrm{m}}$ to different voltages during caffeine application under conditions of $\mathrm{K}^{+}$-channel blockade allowed us to construct current-voltage (I-V) plots with a slope conductance of approximately $220 \mathrm{pS}$ and reversal potential of approximately $0 \mathrm{mV}$ for both species (Figure $1, F$ and $\mathrm{G}$ ). The zero-reversal potential is a typical property of poorly selective $\mathrm{Cx}$ hemichannels. Increasing extracellular $\mathrm{Ca}^{2+}$ 5 -fold significantly increased the slope conductance and shifted the reversal potential rightward in the direction of the increased Nernst potential for $\mathrm{Ca}^{2+}$, indicating substantial $\mathrm{Ca}^{2+}$ flow through 


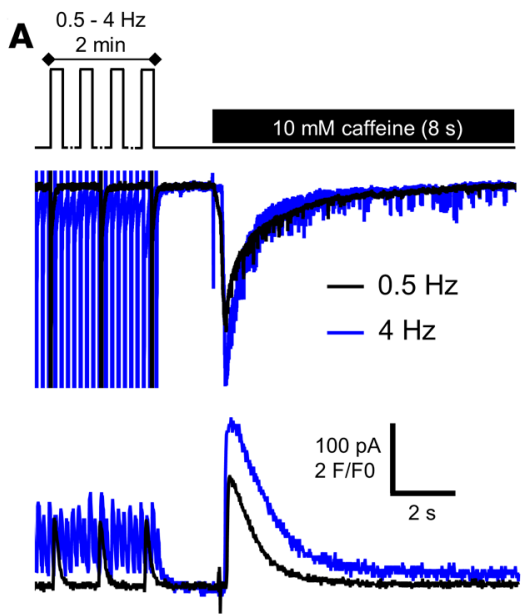

D

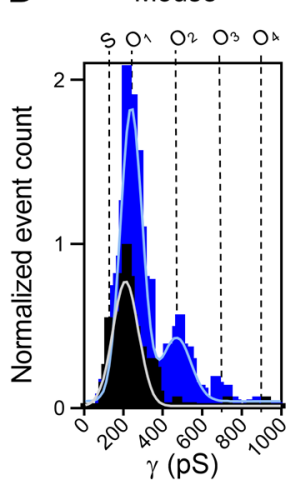

B
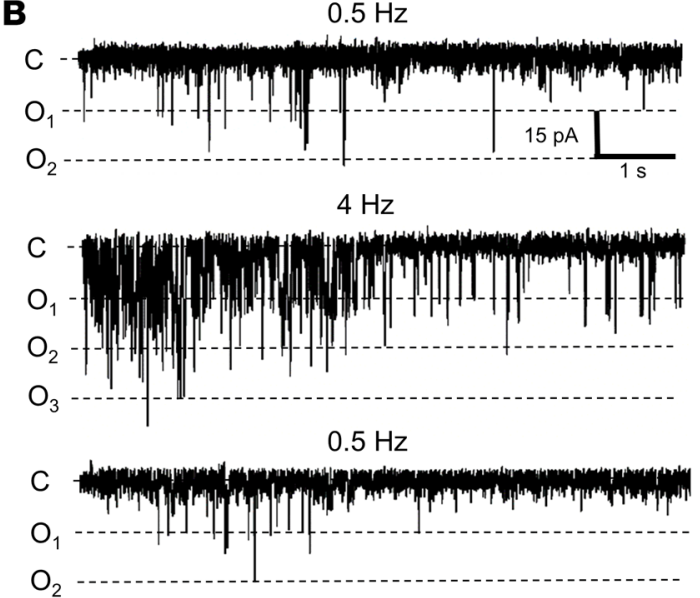

C. Mouse $] P=0.015$

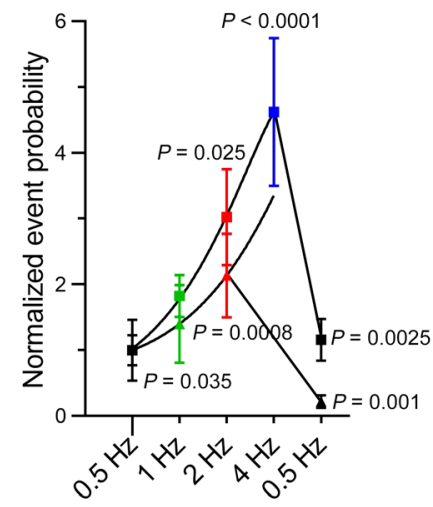

E

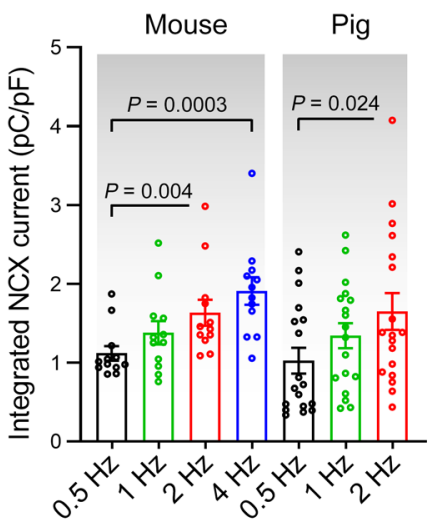

$\mathbf{F}$

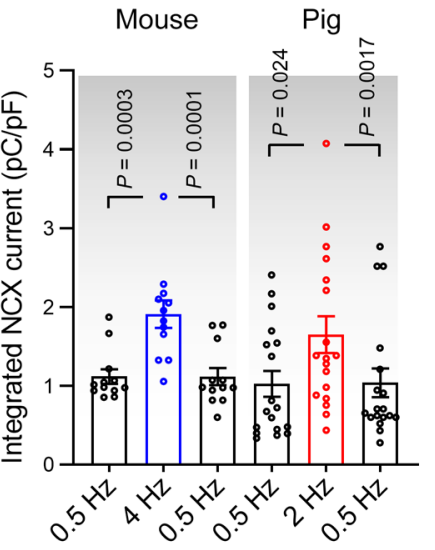

Figure 2. Cx43 hemichannel activation is frequency dependent. (A) Top trace shows experimental protocol: cells were paced for 2 minutes to steady state (mouse $0.5,1,2$, or $4 \mathrm{~Hz}$; pig $0.5,1$, or $2 \mathrm{~Hz}$ ) followed by caffeine superfusion. Middle and bottom traces depict current and [Ca $\left.{ }^{2+}\right]_{i}$ signals recorded in a mouse myocyte after 0.5 (black) or $4 \mathrm{~Hz}$ (blue) pacing. (B) Unitary current example traces after 0.5 and $4 \mathrm{~Hz}$ including reversibility (NCX currents subtracted). (C and $\mathbf{D})$ Summary graph and transition histograms indicate significant and reversible increase in unitary current event probability with increasing pacing frequency $\left(\mathrm{N} / n_{\text {mouse }}=4 / 12, \mathrm{~N} / n_{\text {pig }}=7 / 19\right)$. $P$ values compare to $0.5 \mathrm{~Hz}$ or to $2 / 4 \mathrm{~Hz}$ (nested 1-way ANOVA). (E) Summary dot plot depicting significant increase in SR $\mathrm{Ca}^{2+}$ content with increasing frequency, as determined by integrating NCX current during caffeine (nested 1-way ANOVA; $N / n_{\text {mouse }}=4 / 12, N / n_{\text {pig }}=7 / 19$ ). (F) Summary dot plot depicting reversible increase in SR Ca ${ }^{2+}$ content with increasing frequency (nested 1-way ANOVA; $N / n_{\text {mouse }}=4 / 12, N / n_{\text {pig }}=7 / 19$ ).

the channel (Figure $1 \mathrm{G})$. When a caffeine challenge was given at positive $\mathrm{V}_{\mathrm{m}}$ instead of $-70 \mathrm{mV}$, the short, approximately $8 \mathrm{~ms}$ unitary opening events attained a strongly prolonged character (100-120 $\times$ increase in open time), resembling the long duration hemichannel openings observed previously (refs. 3, 4, 6, Figure 1F, and Supplemental Figure 2D). Unitary current properties and interspecies differences are summarized in Supplemental Table 6.

To confirm the $\mathrm{Cx} 43$ hemichannel origin of the $\mathrm{Ca}^{2+}$ releaseinduced unitary currents, we used a combination of pharmacological and genetic approaches targeting $\mathrm{Cx} 43$ (Figure 1, H and I). Cx43 knockdown by approximately 80\% (Supplemental Figure 5, A-D) strongly and significantly reduced unitary current activities. We additionally tested a set of $\mathrm{Cx} 43$ targeting peptides that interfere with hemichannel function (Figure 1, H and I and Supplemental Figure 6A; for peptide administration and concentrations, see Methods). Gap19 significantly reduced unitary current activities, whereas the inactive mutant (4) Gap19 $9^{1130 \mathrm{~A}}$ had no effect. Conversely, the hemichannel opening enhancer СT9 $(5,24)$ significantly increased unitary currents. We screened for other chan- nels with a similar biophysical profile such as Panx1, TRPP2, and TRPP5 $(25,26)$. Firstly, ventricular Panx1 and TRPP2/5 protein levels were unchanged or undetected in $\mathrm{Cx} 43^{\mathrm{Cre}-\mathrm{ER}(\mathrm{T}) / \mathrm{f}}$ ventricles (Supplemental Figure 5, E-H). Additionally, the pannexin-1 targeting peptide ${ }^{10} \mathrm{Panx} 1$ did not affect unitary currents (Figure 1I). None of the peptides or experimental conditions affected SR $\mathrm{Ca}^{2+}$ content, as determined by integrating the NCX current during caffeine superfusion (Supplemental Figure 6B).

Cx43 hemichannel opening is modulated by stimulation frequen$c y$ and enhanced during $\beta$-adrenergic stimulation. It is well known that cardiomyocyte responses to repeated electrical stimulation or $\beta$-adrenergic stimulation occur in part through alterations in $\left[\mathrm{Ca}^{2+}\right]_{\mathrm{i}}$ signaling and $\mathrm{SR} \mathrm{Ca}^{2+}$ content (20). We thus verified whether $\mathrm{Cx} 43$ hemichannel activity was influenced by increasing stimulation frequency and $\beta$-adrenergic activation. $\mathrm{Ca}^{2+}$ release-induced unitary current activity significantly and reversibly increased when increasing the stimulation frequency before caffeine application ( 0.5 to $4 \mathrm{~Hz}$ range in mouse and 0.5 to $2 \mathrm{~Hz}$ range in pig, Figure $2, \mathrm{~A}-\mathrm{D})$. The increase was proportional to changes in the ampli- 
A
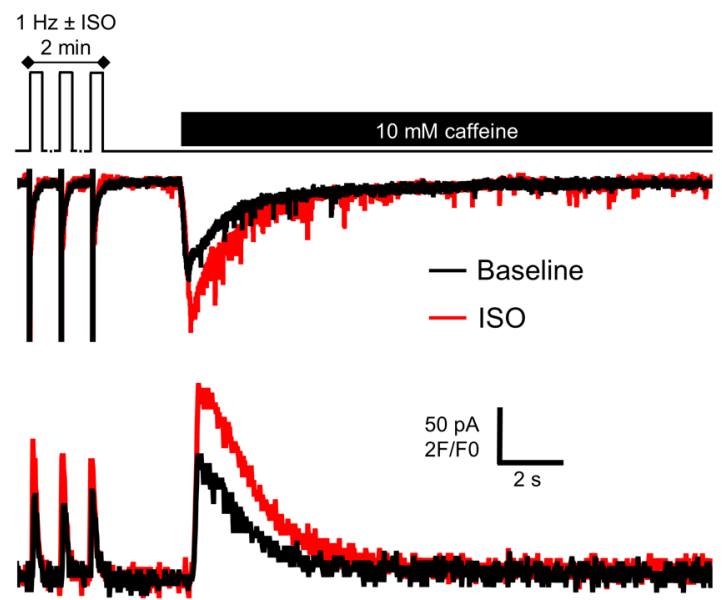

C

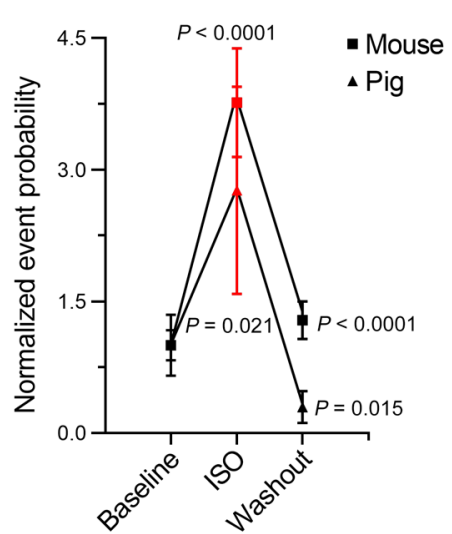

D

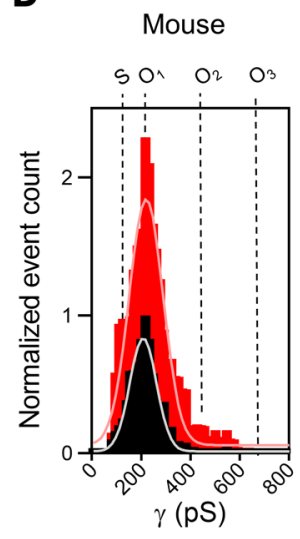

B

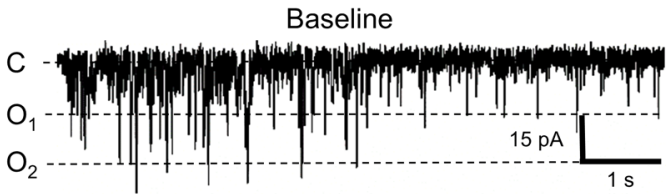

ISO

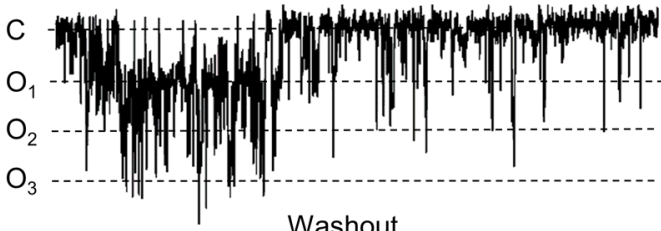

Washout

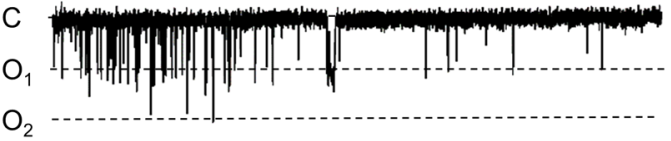

Figure 3. Cx43 hemichannel activation is modulated by $\boldsymbol{\beta}$-adrenergic stimulation. (A) Top trace shows experimental protocol: $\beta$-adrenergic stimulation $(1 \mu \mathrm{mol} / \mathrm{L}$ isoproterenol for mouse; $10 \mathrm{nmol} / \mathrm{L} \mathrm{pig})$ was applied when pacing frequency was $1 \mathrm{~Hz}$. Middle and bottom traces depict current and [Ca $\left.{ }^{2+}\right]_{i}$ signals recorded in mouse following $1 \mathrm{~Hz}$ pacing without (black) or with isoproterenol (red). (B) Unitary current example traces in the absence or presence of isoproterenol, including washout (NCX currents subtracted). (C and D) Summary graph and transition histograms indicate significant and reversible increase in unitary current event probability with isoproterenol compared with baseline (nested 1-way ANOVA; $N / n_{\text {mouse }}=20 / 49, N / n_{\text {pig }}=8 / 20$ ). (E) Summary dot plot illustrating reversible increase in SR $\mathrm{Ca}^{2+}$ content with isoproterenol compared with baseline (nested 1 -way ANOVA; $N / n_{\text {mouse }}=20 / 49, N / n_{\text {pig }}=8 / 20$ ).

tude of the caffeine-induced SR Ca ${ }^{2+}$ release (Figure 2, $\mathrm{E}$ and $\mathrm{F}$ ). Moreover, $\beta$-adrenergic stimulation by superfusion of isoproterenol (ISO) during the 2-minute train at $1 \mathrm{~Hz}$ significantly increased unitary current activities (Figure 3, A-D), in line with concurrent changes in $\mathrm{SR} \mathrm{Ca}^{2+}$ content (Figure 3E).

$C x 43$ associates with dyads at the intercalated disc where microdomain activation of $\mathrm{C} x 43$ hemichannels occurs. Since $\mathrm{Cx} 43$ hemichannels are activated by a subsarcolemmal increase in $\mathrm{Ca}^{2+}$ during caffeine-induced SR $\mathrm{Ca}^{2+}$ discharge, we hypothesized that these channels would intimately associate with cardiac dyads at the intercalated disc. We used single-molecule light microscopy (SMLM) by performing stochastical optical reconstruction microscopy (STORM) to map Cx43 distribution to dyadic nanodomains in single cardiomyocytes and cardiomyocyte cell pairs from the left ventricle of a mouse. We first used immunolabeling of $\mathrm{Cx} 43$ in combination with an SR marker (RyR2, junctophilin-2 [JPH2] or total phospholamban [tPLN]) or a sarcolemmal membrane marker (pore-forming subunit of the L-type $\mathrm{Ca}^{2+}$ channel [Cav1.2], NCX, or caveolin-3 [Cav3]). All markers showed the largest cluster density and cluster size at the cell ends of single cardiomyocytes and at the intercalated discs of cardiomyocyte cell pairs compared with the lateral surface or cell interior (Supplemental Figure 7, A-C). By contrast, the Z-disc protein $\alpha$-actinin - used as a negative control - did not follow such distribution and showed highest cluster density and size in the cell interior (Supplemental Figure 7C). Distance analysis revealed that 30\% to 50\% of the studied markers occurred within approximately $20 \mathrm{~nm}$ of $\mathrm{Cx} 43$ at the cell ends of single cardiomyocytes or at the intercalated discs of cardiomyocyte cell pairs (Supplemental Figure 7D). To exclude random association of $\mathrm{Cx} 43$ to these overtly abundant markers, we performed stochastic simulations on these data to determine the interaction factor, which expresses the relation of the experimentally observed overlap versus the probability for random overlap (interaction factor ImageJ plugin, ref. 27). Cx43 colocalized to all markers with an interaction factor higher than 0.6 , indicating that the observed overlap was deterministic rather than random (Supplemental Figure 7, E and F).

We used a triple staining of Cav1.2, RyR2, and Cx43 to directly map the relation of $\mathrm{Cx} 43$ to dyads (based on Cav1.2 and RyR2 clusters occurring $<250 \mathrm{~nm}$ from each other; refs. 28, 29 and 
A
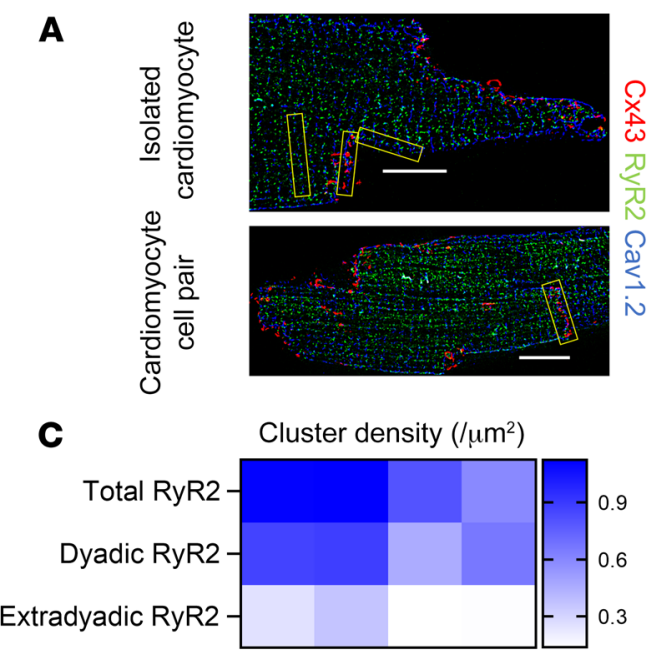

No. RyRs/cluster

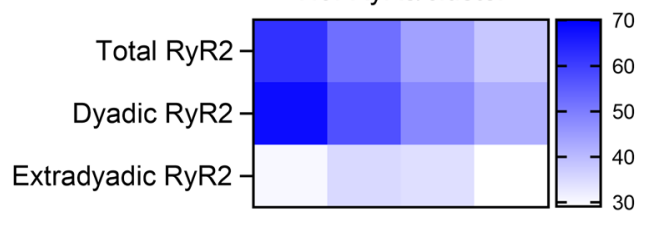

Colocalization with Cx43 (\%)

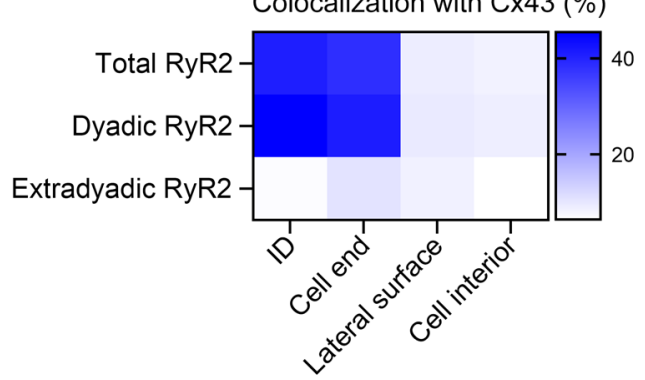

E

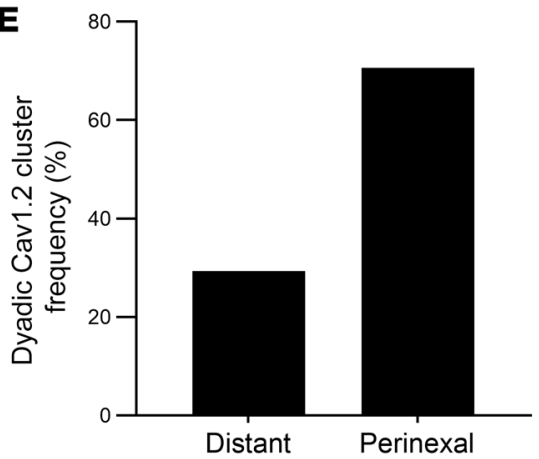

B

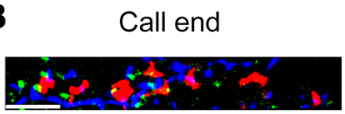

Lateral surface

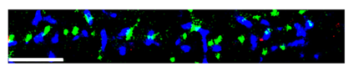

D

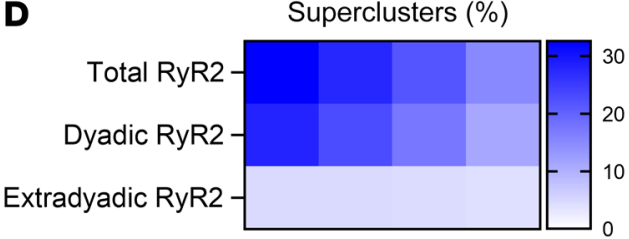

No. RyRs/supercluster
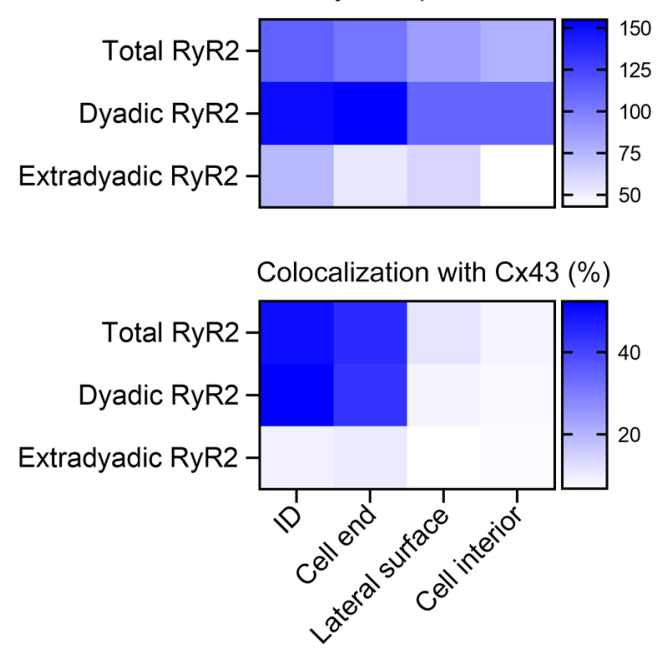

$\mathbf{F}$

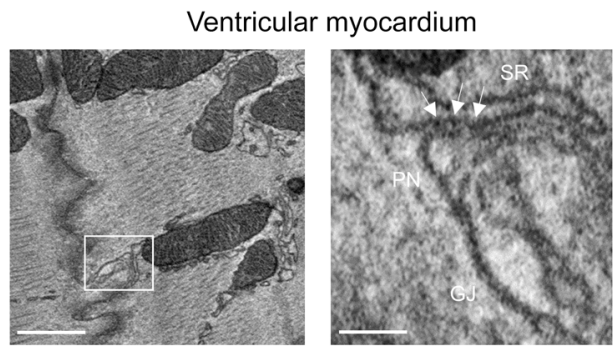

Figure 4. Cx43 colocalizes with large dyadic RyR2 superclusters and forms microdomains at the perinexus. (A) 2D SMLM images of a murine cardiomyocyte (top) and cardiomyocyte cell pair (bottom), triple stained for Cx43 (red), RyR2 (green), and Cav1.2 (blue). Scale bar: $10 \mu \mathrm{m}$. (B) Straightened region of interest (from yellow boxes in A) of Cx43, RyR2, and Cav1.2 at different subcellular domains. Scale bar: $2 \mu \mathrm{m}$. (C) Heatmap of RyR2 cluster density, number of molecules, and colocalization with Cx43 at different subcellular domains ( $n=5, n=42$ single cardiomyocytes, 16 cardiomyocyte cell pairs). RyR2 clusters were classified as dyadic or extradyadic based on the proximity of Cav1.2 clusters, RyR2 clusters occurring less than $250 \mathrm{~nm}$ from a Cav1.2 cluster were categorized as dyadic. (D) Heatmap of RyR2 supercluster abundance, size, and colocalization with Cx43 at different subcellular domains ( $n=5, n=42$ single cardiomyocytes, 16 cardiomyocyte cell pairs). (E) Relative localization overview in left ventricular mouse cardiomyocyte cell pairs. Dyadic Cav1.2 clusters were categorized as perinexal or distant based on edge distance $200 \mathrm{~nm}$ or less or greater than $200 \mathrm{~nm}$ from edge of Cx43 cluster, respectively ( $n=5$, $n=16$ cardiomyocyte cell pairs). (F) EM images of an SR cistern forming a dyadic cleft at the perinexus in mouse ventricular myocardium. Left image shows an EM overview of a murine ventricular intercalated disc. Scale bar: $500 \mathrm{~nm}$. White box is enlarged on the right. Arrows indicate electron dense particles, likely ryanodine receptors. Scale bars: $100 \mathrm{~nm}$. Pn, perinexus. 
A
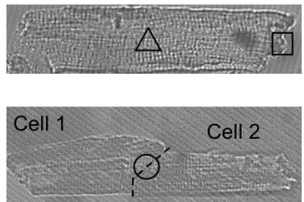

$\square \quad$ Cell end

$\triangle \quad$ Lateral surface

Intercalated disc
B

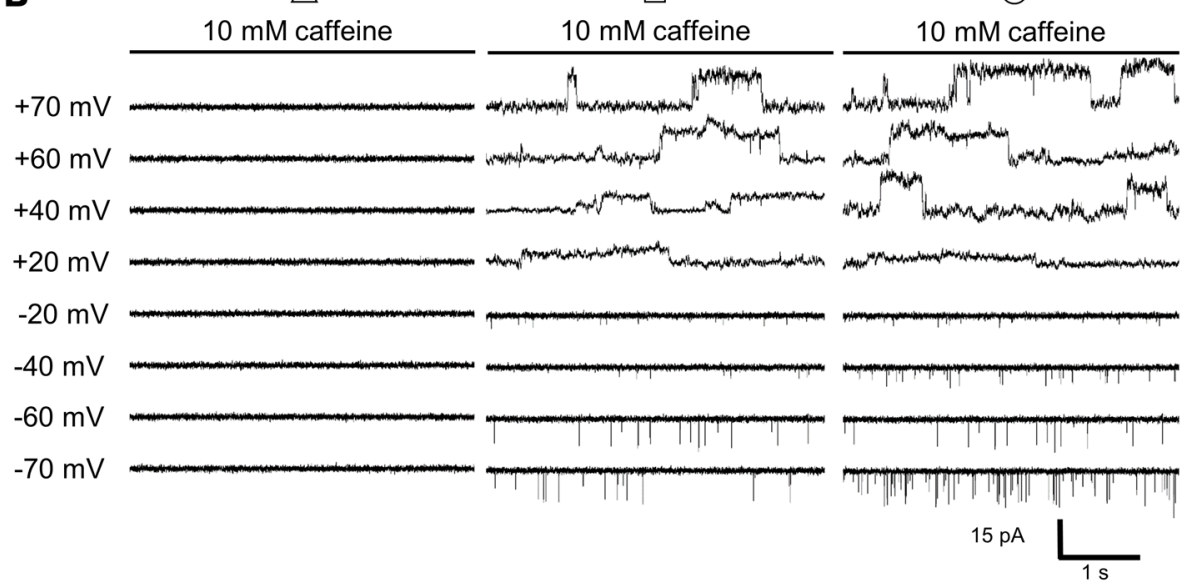

C

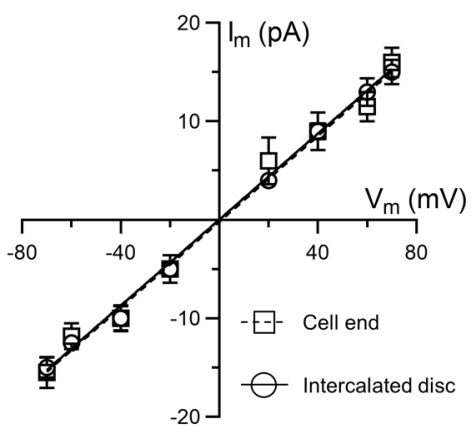

Pig

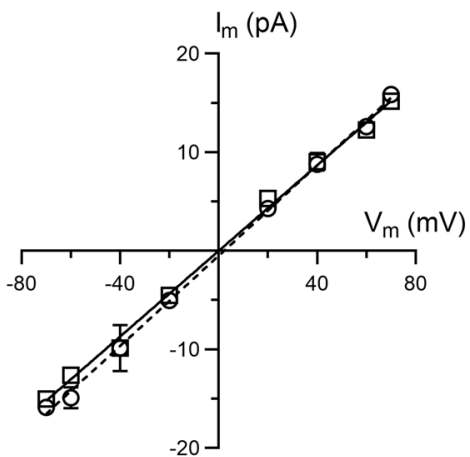

$\mathbf{E}$

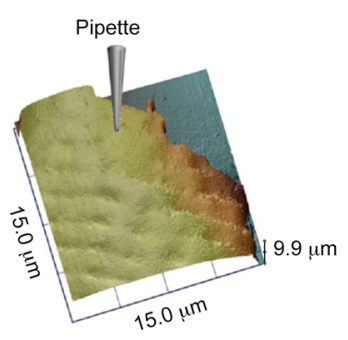

D
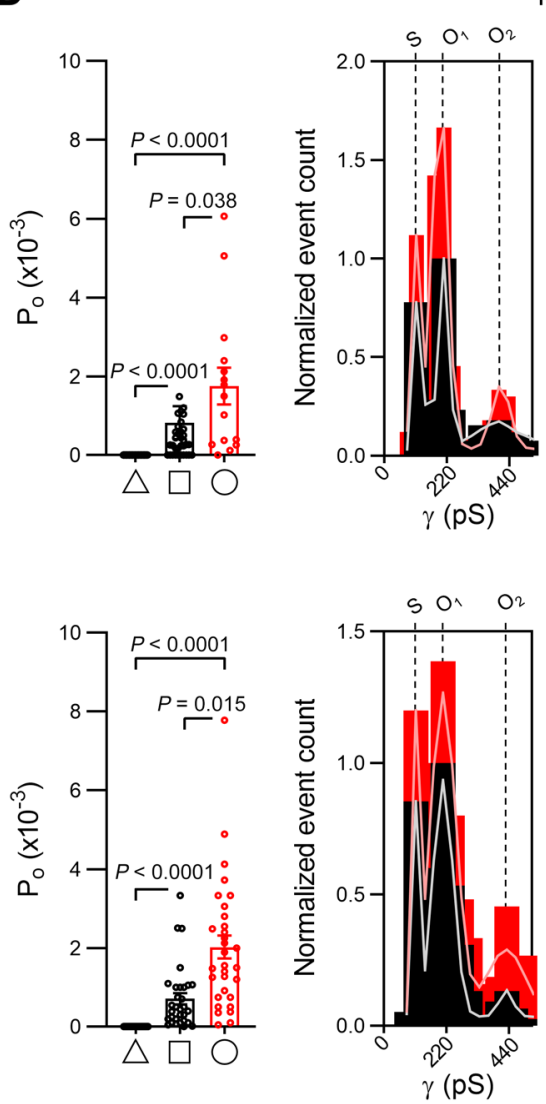

Mouse

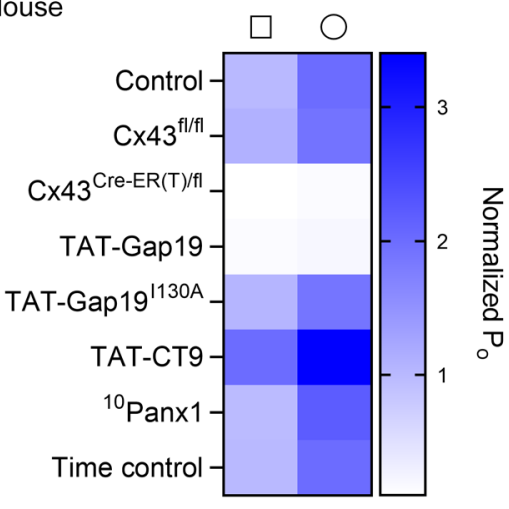

Pig

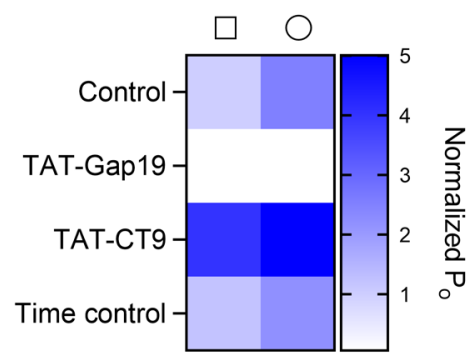

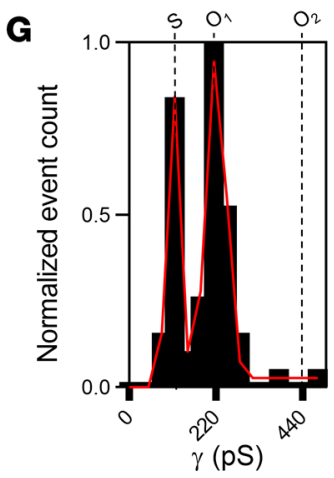

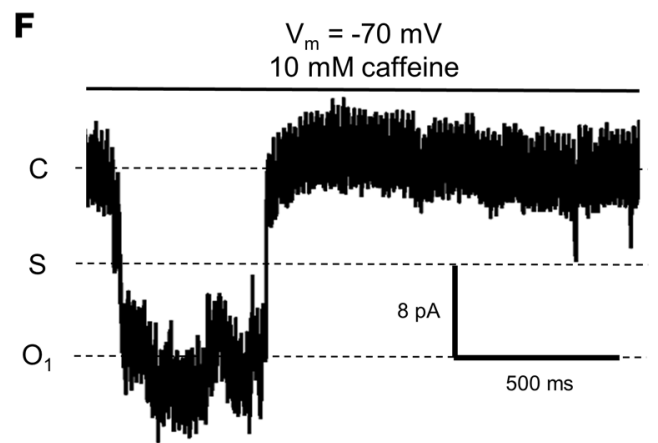


Figure 5. Discrete sites of $\mathrm{C} \times 43$ hemichannel activation at the intercalated disc during $\mathrm{Ca}^{2+}$ release from the sarcoplasmic reticulum. (A) Transmitted light images of a single cardiomyocyte (top) and cardiomyocyte cell pair (bottom). Triangle, square, and circle symbols indicate cell-attached macropatch $\left(R_{p}=\sim 2 \mathrm{M} \Omega, \sim 2 \mu \mathrm{m}\right.$ pipette inner diameter) recording positions at the lateral membrane and cell end of single cardiomyocytes and intercalated disc of cardiomyocyte cell pairs, respectively. (B) Example traces showing single channel currents recorded at the lateral membrane, cell end, or intercalated disc. Traces recorded in mouse cardiomyocytes during caffeine superfusion ( $10 \mathrm{mM}, 8$ seconds) at indicated membrane potentials. (C) IV plots depicting linear current-voltage relationship with slope conductance of approximately $220 \mathrm{pS}$ and $\mathrm{E}_{\text {rev }} \approx 0 \mathrm{mV}\left(\mathrm{N} / \mathrm{n}_{\text {mouse }}=5 / 10-18\right.$ patches per condition, $N / n_{\text {pig }}=5 / 15-20$ patches per condition). (D) Summary dot plots and transition histograms indicate recording of approximately $220 \mathrm{pS}$ single channel currents at the cell end of single cardiomyocytes, but not at the lateral membrane, with significantly increased open probability at the intercalated disc of cardiomyocyte cell pairs. Comparative statistics with nested 1-way ANOVA. Heatmap summarizes single channel open probability at the cell end or at the intercalated disc under conditions of Cx43 knockdown or in the presence of TAT-Gap19, inactive TAT-Gap19130A, TAT-CT9, or ${ }^{10} \mathrm{Panx} 1\left(\mathrm{~N} / \mathrm{n}_{\text {mouse }}=5 / 10-18\right.$ patches per condition, $N / n_{\text {pig }}=$ 5/15-20 patches per condition). (E) SICM-generated membrane topology of the cell end of a mouse left ventricular cardiomyocyte. Pipette indicates the recording position distally of the last Z-line. (F) Example trace recorded at $-70 \mathrm{mV}$ during caffeine superfusion. (C) Transition histogram from all experiments $(n=5, n=35)$ showing a fully open state at approximately 220 $\mathrm{pS}$ and a substate at approximately $110 \mathrm{pS}$.

Figure 4, A and B). We found that approximately $80 \%$ of RyR2 associated in dyads and that dyadic RyR2 clusters were larger than their extradyadic counterparts. Both dyadic and extradyadic RyR2 cluster densities and number of molecules were largest at the cell ends of single cardiomyocytes and at the intercalated discs of cardiomyocyte cell pairs compared with the lateral surface or cell interior (Figure 4C). Only a small fraction of extradyadic RyR2 clusters occurred within approximately $20 \mathrm{~nm}$ of $\mathrm{Cx} 43$, whereas approximately $50 \%$ of dyadic RyR 2 colocalized with $\mathrm{Cx} 43$ (Figure 4C). Recent work has shown that closely localized RyR clusters, at less than $100 \mathrm{~nm}$ edge-to-edge distance, may act cooperatively as superclusters to generate $\mathrm{Ca}^{2+}$ signals (30). These RyR superclusters preferentially organized in dyads and occurred more frequently at the cell ends of single cardiomyocytes and at the intercalated discs of cardiomyocyte cell pairs compared with the lateral surface or cell interior (Figure 4D). Superclusters at these sites contained more RyRs (Figure 4D). Overall, dyadic superclusters formed the majority of structures that colocalized with $\mathrm{Cx} 43$.

Next, we used a relative localization algorithm $(31,32)$ to categorize dyadic Cav1.2 clusters at the intercalated discs of cardiomyocyte cell pairs as either located in the perinexus (based on the signal overlapping with $\mathrm{Cx} 43$ and extending within $200 \mathrm{~nm}$ of $\mathrm{Cx} 43$ clusters) or distant from $\mathrm{Cx} 43$ (based on signal extending beyond $200 \mathrm{~nm}$ of $\mathrm{Cx} 43$ clusters). We found that approximately $80 \%$ of Cav1.2 clusters occurred in dyads; approximately $42 \%$ of these clusters overlapped with $\mathrm{Cx} 43$ and another approximately $28 \%$ occurred adjacent to $\mathrm{Cx} 43$, resulting in approximately $70 \%$ of dyads at the intercalated disc occurring in the perinexal nanodomain where $\mathrm{Cx} 43$ hemichannels are known to reside (Figure $4 \mathrm{E}$ and Supplemental Figure 8A). Cx43 and dyadic Cav1.2 clusters overlapped only partially, confirming association at the edge of $\mathrm{Cx} 43$ clusters (Supplemental Figure 8B). In line with these observations, electron microscopy of murine cardiac ventricular tissue revealed cleft formation of SR cisterns with the perinexal nanodomain where $\mathrm{Cx} 43$ hemichannels reside (Figure 4F, Supplemental Video 1 and 2, and refs. 33, 34). Based on the protein structure of $\mathrm{Cx} 43$ channels (35), the distance to nearest neighbor in cardiac gap junction plaques (36), and the lower density of $\mathrm{Cx} 43$ in the perinexus (37), we calculated that perinexal dyads or dyads at the cell end may contain 1-2 Cx43 hemichannels per dyad (Supplemental Figure 9).

To provide a functional correlate to these structural observations, we used the macropatch technique (38) to map single Cx43 hemichannel activity at discrete sarcolemmal microdomains. During caffeine-induced SR $\mathrm{Ca}^{2+}$ release, we observed single-channel currents with a conductance of approximately $220 \mathrm{pS}$ at the cell ends of single cardiomyocytes and intercalated discs of cardiomyocyte cell pairs. One-third of patches at these sites had currents, with currents being absent in lateral membranes (Figure 5, A-D and Supplemental Table 7). Open probability was highest in cardiomyocyte cell pairs. Events were inhibited by TAT-Gap19, enhanced by TAT-CT9, and abolished in cells not expressing Cx43. ${ }^{10}$ Panx1 and inactive TAT-Gap19 ${ }^{1130 A}$ had no effect (Figure 5D). We used scanning ion conductance microscopy (SICM) to map membrane topology at the cell end and recorded approximately $220 \mathrm{pS}$ caffeine-induced unitary current activity (including $\sim 110 \mathrm{pS}$ substate) in nanopatches $\left(\mathrm{R}_{\mathrm{p}} \sim 30 \mathrm{M} \Omega, \sim 300\right.$ $\mathrm{nm}$ pipette internal diameter) just distally of t-tubules at the start of the intercalated disc (Figure 5, E-G). Patches contained 1 to 2 active channels, in line with our predictions (Supplemental Figure 9 and Supplemental Table 7).

Cx43 hemichannels modulate diastolic $\mathrm{Ca}^{2+}$ release during adrenergic stimulation and associated arrhythmogenic afterdepolarizations. Since highly conductive and $\mathrm{Ca}^{2+}$-permeable hemichannels are localized near dyads at the intercalated disc, it is conceivable that they could contribute to diastolic SR $\mathrm{Ca}^{2+}$ release itself (11). To investigate this, we exposed the cardiomyocytes to fast pacing and $\beta$-adrenergic stimulation without caffeine and recorded spontaneous SR $\mathrm{Ca}^{2+}$ release events and currents occurring after such stimulation (Figure 6A). Additionally, we monitored $V_{m}$ changes after switching to current clamp conditions following the same stimulation protocol (Figure 6B). Compared with baseline, diastolic $\mathrm{Ca}^{2+}$ release, accompanying currents, and afterdepolarizations became prominent after fast pacing and ISO exposure (Figure 6C). Gap19 and Cx43 knockdown reduced the number of diastolic $\mathrm{Ca}^{2+}$ release events and associated currents; CT9 enhanced these events and Gap191130A had no effect (Figure 6D). Accordingly, DADs and triggered action potentials after a period of fast pacing and ISO stimulation (Figure 6, B and C) were significantly reduced by TAT-Gap19, not affected by TAT-Gap19 ${ }^{\text {I130A }}$, and enhanced by TAT-CT9 (Figure 6D).

We verified whether the $\mathrm{Cx} 43$ targeting peptides or $\mathrm{Cx} 43$ knockdown would perhaps affect the properties of the global $\left[\mathrm{Ca}^{2+}\right]_{\mathrm{i}}$ transient. This was not the case, indicating absence of gross effects of these interventions on cardiomyocyte global $\mathrm{Ca}^{2+}$ homeostasis (Supplemental Figure 10). Our next approach was to further scrutinize the occurrence of hemichannel activity after adrenergic stimulation in the time window of macroscopic NCX currents associated with spontaneous $\mathrm{Ca}^{2+}$ release. We 
A

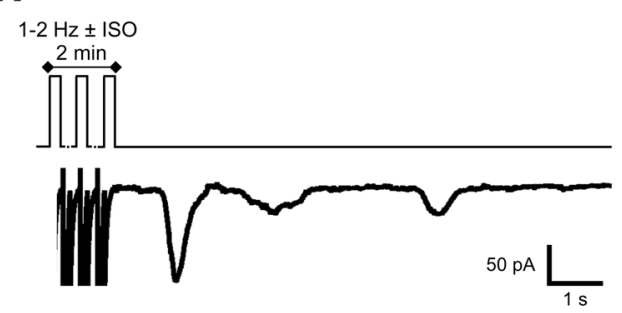

MnM $\sim^{1 \in F_{0}}$
B Voltage clamp

$1-2 \mathrm{~Hz} \pm$ ISO

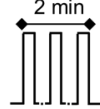

C
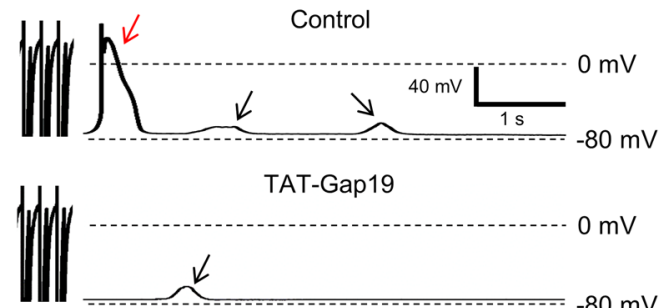

TAT-Gap19
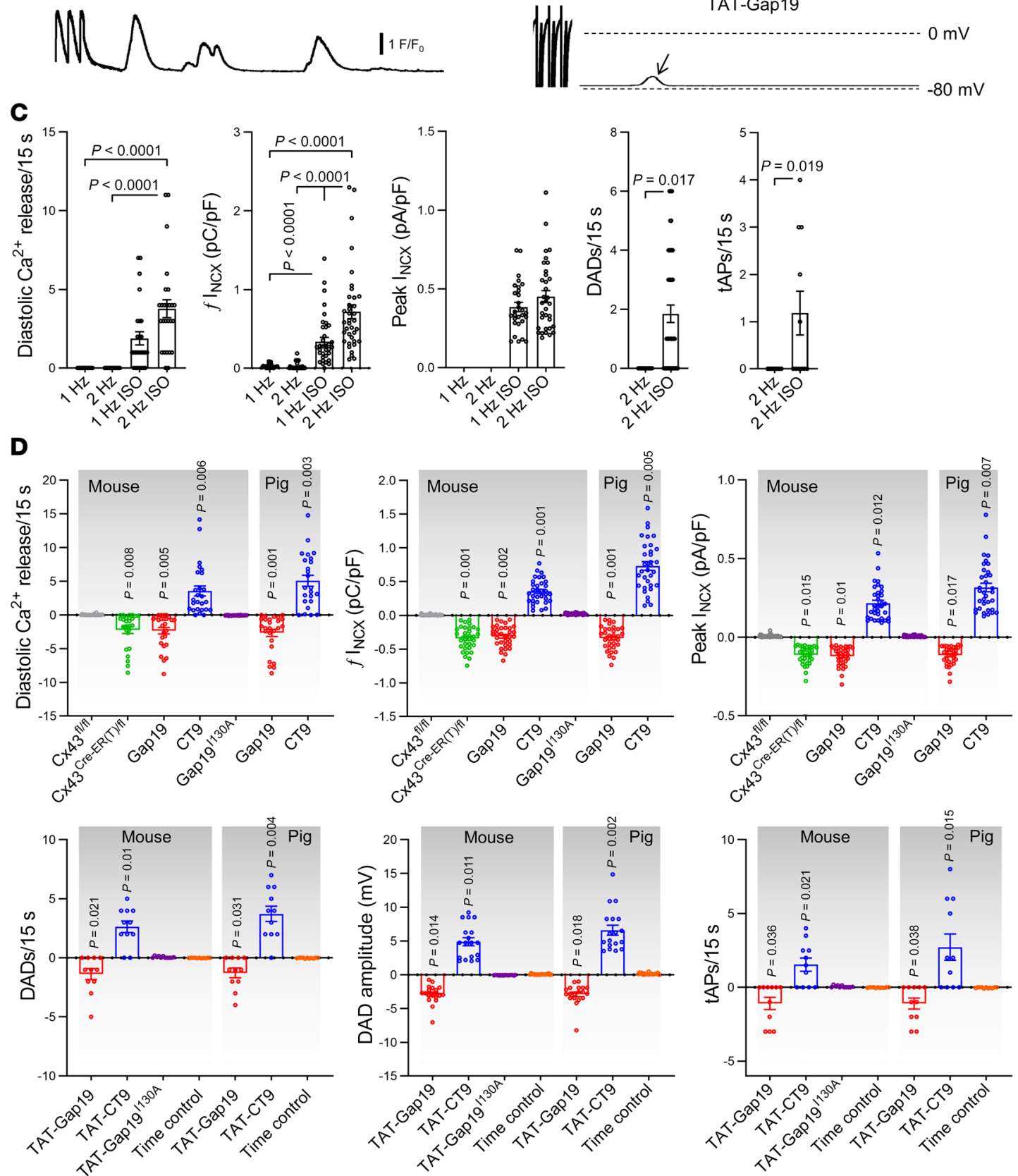
Figure 6. Cx43 hemichannel opening during adrenergic stimulation modulates spontaneous $\mathrm{Ca}^{2+}$ release from the sarcoplasmic reticulum and arrhythmogenic afterdepolarizations. (A) Freshly isolated mouse and pig left ventricular cardiomyocytes were subjected to voltage clamp experiments while $\left[\mathrm{Ca}^{2+}\right]_{i}$ was simultaneously monitored. Top trace shows experimental protocol: cells were paced to steady state for 2 minutes at $1 \mathrm{~Hz}$ and then clamped to $-70 \mathrm{mV}$. Middle and bottom traces depict resulting current and $\left[\mathrm{Ca}^{2+}\right]_{i}$ signals: final 3 paced $\mathrm{Ca}^{2+}$ transients and accompanying currents followed by 15 -second rest period showing spontaneous diastolic $\mathrm{Ca}^{2+}$ release with resulting NCX current. Protocols were repeated at $2 \mathrm{~Hz}$ with and without isoproterenol. Example traces were recorded in pig cardiomyocytes. (B) In a subset of experiments, we switched to current clamp mode following steady-state pacing in voltage clamp. Example traces, recorded in the same pig cardiomyocyte, without and with TAT-Gap19 were recorded in current clamp mode following 2-minute pacing to steady state at $2 \mathrm{~Hz}$ with isoproterenol (in voltage clamp mode). Black arrows indicate DADs, red arrow indicates a triggered action potential. (C) Summary dot plots (nested 1-way ANOVA; $N / n_{\text {mouse }}$ $=23 / 75$ for voltage clamp experiments and $N / n_{\text {mouse }}=5 / 45$ for current clamp experiments) illustrating increased frequency and amplitude of diastolic $\mathrm{Ca}^{2+}$ release with increased resulting NCX current and membrane depolarization during adrenergic stimulation ( $2 \mathrm{~Hz}+$ ISO) compared with baseline. tAP, triggered action potential. Similar results were obtained in pig cardiomyocytes (not shown). (D) Summary data showing the impact of different interventions on diastolic $\mathrm{Ca}^{2+}$ release and resulting NCX currents and membrane depolarization (nested 1-way ANOVA; $N / n_{\text {mouse }}=$ $5-11 / 15-24$ per condition, $N / n_{\text {pig }}=5 / 15-20$ per condition). Values reported as differences from the control condition.

found that approximately $220 \mathrm{pS}$ unitary current events occurred not only during the NCX current (superimposed on it, see Figure $7 \mathrm{~A}$ ) but also preceding it (Figure 8A; corresponding conductance histograms are shown in Figures $7 \mathrm{~B}$ and $8 \mathrm{~B}$ respectively). Event probabilities before and during NCX were comparable (compare Figures $7 \mathrm{C}$ with $8 \mathrm{C}$ ) and strongly increased by pacing and ISO. Unitary current activity during adrenergic stimulation was robustly associated with larger $\mathrm{Ca}^{2+}$ transients, larger $\mathrm{SR} \mathrm{Ca}^{2+}$ content, and increased spontaneous $\mathrm{Ca}^{2+}$ release (Supplemental Figure 11). Genetic ablation and pharmacological tools had effects, as observed in the caffeine-triggered responses (Figures 7D and 8D; compare to Figure 1I). As expected, charge transfer associated with these unitary hemichannel events was significantly smaller compared with charge transfer linked to the NCX current during spontaneous $\mathrm{Ca}^{2+}$ release (Figure 7E).

As a control experiment, we tested whether Gap19, CT9, and their TAT versions affected $\mathrm{Ca}^{2+}$ release via RyR2. To this end, we challenged RyR2-overexpressing HEK293 cells (39) with caffeine and quantified $\mathrm{Ca}^{2+}$ signals in the presence or absence of peptide. Overall, peptides did not influence caffeine-induced $\mathrm{Ca}^{2+}$ signals, except for acute TAT-CT9 exposure, which significantly increased $\mathrm{Ca}^{2+}$ release at $5 \mathrm{mM}$ caffeine but not at 0.4 $\mathrm{mM}$; no effect was observed when TAT-CT9 was preincubated (Supplemental Figure 12).

$\mathrm{Cx} 43$ hemichannel depolarizing current, microdomain $\mathrm{Ca}^{2+}$ entry, and RyR coupling underlie hemichannel-associated triggered activities. To better understand these observations, we performed order of magnitude calculations within a mathematical framework (see Mathematical Framework in Supplemental Methods) incorporating the present unitary current and STORM imaging data. We reasoned that open hemichannels pass inward current and facilitate $\mathrm{Ca}^{2+}$ entry into the cell. The direct contribution of depolarizing current through a single hemichannel was determined to be approximately $1.6 \mathrm{mV}$ (at $-70 \mathrm{mV}$ and $37^{\circ} \mathrm{C}$; Figure 9A). We further estimated the hemichannel $\mathrm{Ca}^{2+}$ current to be approximately $0.84 \mathrm{pA}$ and approximately $1.46 \mathrm{pA}$ for $1.0 \mathrm{mM}$ (mouse) and $1.8 \mathrm{mM}$ (pig and human) extracellular $\mathrm{Ca}^{2+}$, respectively, corresponding to a $\mathrm{Ca}^{2+}$ entry rate of approximately $4.35 \times$ $10^{-18}$ and approximately $7.57 \times 10^{-18} \mathrm{~mol} \mathrm{Ca}^{2+}$ per second and per open hemichannel, respectively. We calculated that such $\mathrm{Ca}^{2+}$ influxes did not affect global cytosolic $\mathrm{Ca}^{2+}$ (in line with the experimental findings presented in Supplemental Figure 10 and refs. $40-44)$, but resulted in a pronounced elevation of peak $\left[\mathrm{Ca}^{2+}\right]_{\mathrm{i}}$ in the hemichannel-dyad microdomain of approximately 0.81 $\mu \mathrm{mol} / \mathrm{L}$ and $3.44 \mu \mathrm{mol} / \mathrm{L}$, respectively $(1.0$ and $1.8 \mathrm{mM}$ extracellular $\mathrm{Ca}^{2+}$, respectively) (Figure 9B, calculations based on a hemichannel open time $\tau$ of $8 \mathrm{~ms}$ derived from the open time distribution). Such $\mathrm{Ca}^{2+}$ elevation may activate significant NCX current in the order of $0.12 \mathrm{pA} / \mathrm{pF}$ and $0.13 \mathrm{pA} / \mathrm{pF}$, respectively (1.0 and $1.8 \mathrm{mM}$ extracellular $\mathrm{Ca}^{2+}$, respectively), producing approximately $1.28 \mathrm{mV}$ and approximately $1.51 \mathrm{mV}$ depolarization, respectively (for 1.0 and $1.8 \mathrm{mM} \mathrm{Ca}^{2+}$, respectively) (Figure 9, A and C). Thus, during a DAD, where hemichannel opening probability is largest during peak NCX current (Figure 7F), membrane depolarization will amount to approximately $2.88 \mathrm{mV}$ and approximately $3.11 \mathrm{mV}$ per hemichannel (Figure 9A), which is in line with the experimental observation that TAT-Gap19 decreased DAD amplitude by 2.83 $\mathrm{mV}$ (Figure 6D). Conversely, TAT-CT9 increased DAD amplitude by $4.9 \mathrm{mV}$, which may be related to longer opening or occasional stacked hemichannel openings (Figure 6D). Thus, hemichannel opening will increase the DAD peak amplitude, bringing it closer to the threshold for action potential firing.

We further included a 4-state Markov RyR gating model (45) in the hemichannel-dyad microdomain to estimate the effect of hemichannel $\mathrm{Ca}^{2+}$ entry on $\mathrm{Ca}^{2+}$-induced $\mathrm{Ca}^{2+}$ release. This suggested that single hemichannel $\mathrm{Ca}^{2+}$ entry may activate an RyR supercluster at the cell end with a probability of approximately 0.72 and 0.99 (for 1.0 and $1.8 \mathrm{mM}$ extracellular $\mathrm{Ca}^{2+}$, respectively), thus providing a $\mathrm{Ca}^{2+}$ spark (Figure 9A). This $\mathrm{Ca}^{2+}$ spark at the intercalated disc has an approximately 0.99 probability of activating a neighboring RyR cluster; accordingly, $\mathrm{Ca}^{2+}$ entry through a single hemichannel has a respective probability of approximately 0.71 and 0.98 (1.0 and $1.8 \mathrm{mM}$ extracellular $\mathrm{Ca}^{2+}$, respectively) to induce a propagating $\mathrm{Ca}^{2+}$ wave (Figure 9, A, D, and E and ref. 46). Experimentally, we analyzed coupling of $\mathrm{Cx} 43$ hemichannel opening activity to diastolic $\mathrm{Ca}^{2+}$ release and the relation to the site of origin, in particular at the cell end. This analysis revealed that hemichannel currents preceded approximately $50 \%$ of the spontaneous $\mathrm{Ca}^{2+}$ release events after adrenergic stimulation in mice, i.e., somewhat lower than the $71 \%$ predicted; this coupling was fast $(\tau \approx 10 \mathrm{~ms})$ and occurred almost exclusively for waves that started at the cell end (Supplemental Video 4 and Figure 8, A and E) compared with the middle (Supplemental Video 3, Figure 7A, and Figure 8E). $\mathrm{Ca}^{2+}$ waves originating from the cell end had a significantly higher amplitude and larger associated NCX currents compared with those originating in the cell middle (Figure 8F). 
A

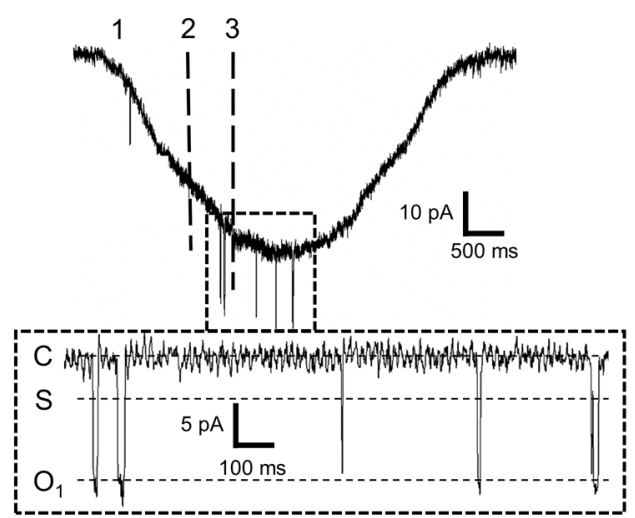

1

2

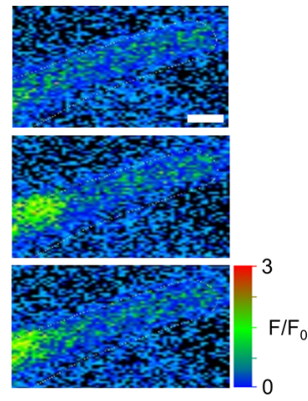

B

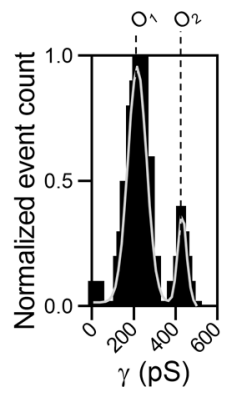

Pig

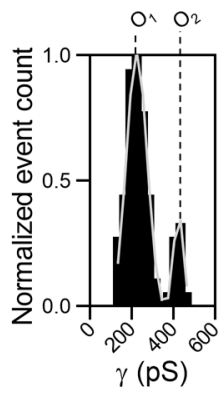

C

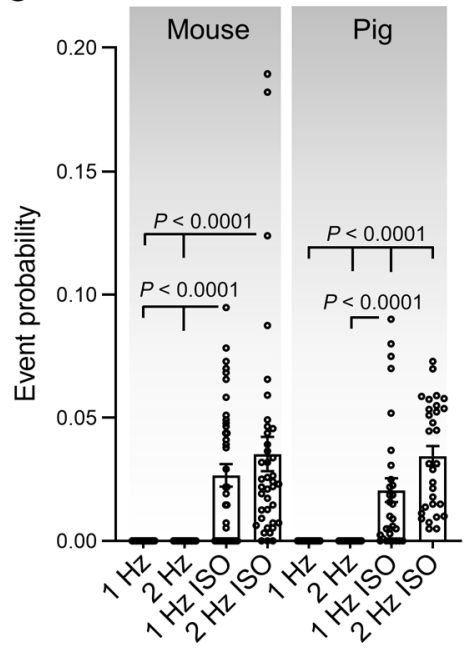

D

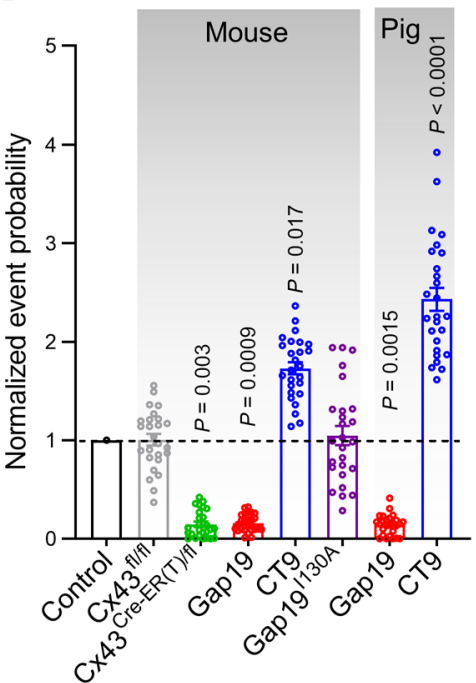

E

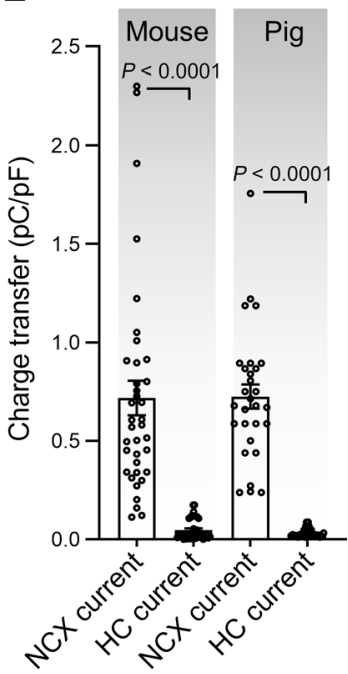

$\mathbf{F}$

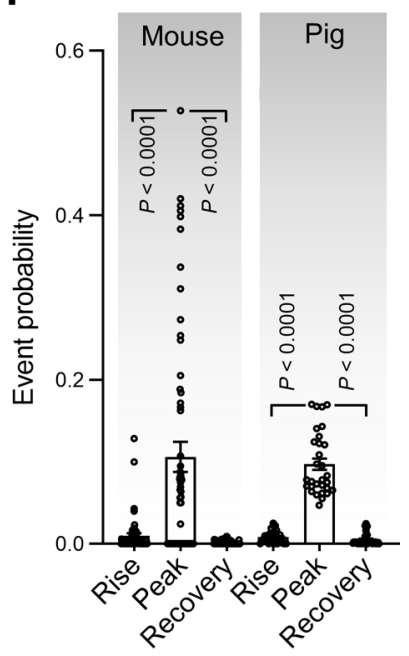

Figure 7. Spontaneous Cx43 hemichannel openings during $\mathbf{C a}^{2+}$ waves. (A) Left, NCX current during spontaneous Ca ${ }^{2+}$ release with superimposed unitary currents. Inset shows detail of unitary current activity. Right, Ca ${ }^{2+}$ images corresponding to time points indicated by dashed lines in left trace. Scale bar: 10 $\mu \mathrm{m}$. (B) Transition histograms of superimposed unitary activity showing approximately $220 \mathrm{pS}$ unitary conductance $\left(N / n_{\text {mouse }}=23 / 75, N / n_{\text {pig }}=10 / 30\right)$. (C) Summary dot plot illustrating increasing unitary current event probability with increasing pacing frequency and with isoproterenol (nested 1-way ANOVA; $\left.\mathrm{N} / \mathrm{n}_{\text {mouse }}=23 / 75, \mathrm{~N} / \mathrm{n}_{\text {pig }}=10 / 30\right)$. (D) Summary data showing the effect of different interventions at $2 \mathrm{~Hz}+\mathrm{ISO}\left(\mathrm{N} / \mathrm{n}_{\text {mouse }}=5-11 / 15-24 \mathrm{per}\right.$ condition, $N / n_{\text {pig }}=$ $5 / 15$ per condition). $P$ values indicate significance compared with control (nested 1-way ANOVA). (E) Summary dot plot depicting relative integrals of NCX and unitary current at $2 \mathrm{~Hz}+\mathrm{ISO}$ (nested $t$ test; $N / n_{\text {mouse }}=23 / 75, N / n_{\text {pig }}=10 / 30$ ). (F) Summary dot plot depicting unitary current event probability during different phases of NCX current induced by spontaneous Ca ${ }^{2+}$ release (nested 1-way ANOVA; $N / n_{\text {mouse }}=23 / 75, N / n_{\text {pig }}=10 / 30$ ). These phases include the rising phase (rise, 10\%-90\%), peak and recovery (90\%-10\%).

Cx43 hemichannels affect cardiac excitability in human heart failure. In human heart failure, $\mathrm{Cx} 43$ expression and distribution change, and we asked the question of whether in those conditions, compared with healthy hearts, $\mathrm{Cx} 43$ hemichannels affect cardiac excitability. We examined explanted hearts from patients with end-stage heart failure; nonfailing rejected donor hearts were used as controls. Patient characteristics are summarized in Supplemental Table 8. Failing hearts were hypertrophic, dilated, and showed severely reduced left ventricular ejection fraction (Supplemental Table 8 and Supplemental Figure 13, A and B). Concurrently, isolated left ventricular cardiomyocytes from failing hearts were hypertrophied compared with controls (Supplemental Figure 13, C and D). In cardiomyocytes from nonfailing human left ventricles, caffeine-induced SR $\mathrm{Ca}^{2+}$ release evoked approximately $220 \mathrm{pS}$ unitary current activity superimposed on the NCX current (Figure 10,
A-C). Unitary events increased with stimulation at higher frequency and were ISO sensitive (Figure 10, D-G), as observed in mice and pig cardiomyocytes (Figure 2). Interestingly, these unitary current activities were more frequent in cardiomyocytes from failing hearts, especially during fast pacing and adrenergic stimulation (Figure 10, D-I) and were inhibited by Gap19 (Figure 10, H and I). In nonfailing human cardiomyocytes, approximately 220 pS unitary current activity could only be recorded at the cell ends of single cardiomyocytes or the intercalated disc of cardiomyocyte cell pairs; in failing cardiomyocytes, unitary current activity was also recorded at the lateral membrane of single cardiomyocytes and cardiomyocyte cell pairs (Figure 11, A-D). In heart failure, patches contained more channels with higher open probability (Figure 11, E and F). In nonfailing and failing cardiomyocytes, these unitary currents were blocked by subsequent application of TAT-Gap19 (Figure 11, D and F). 
A

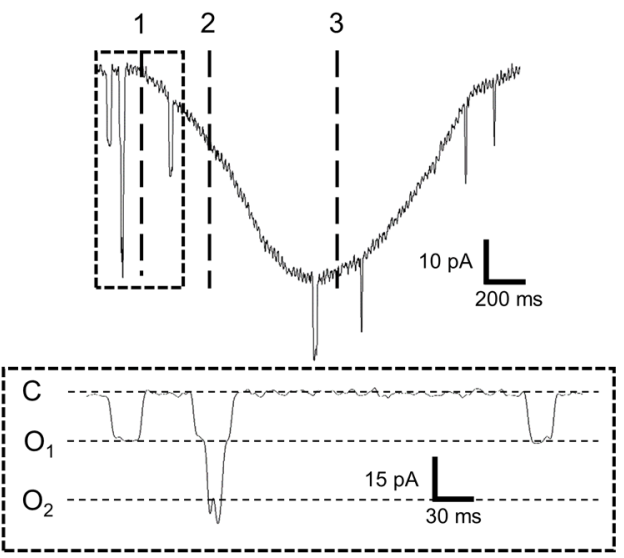

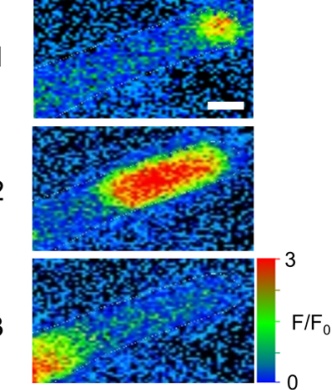

B

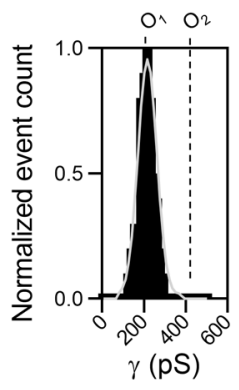

Pig

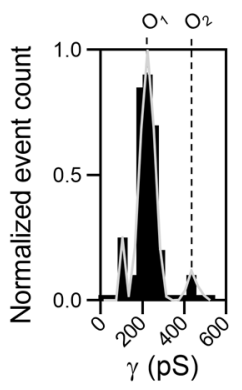

C

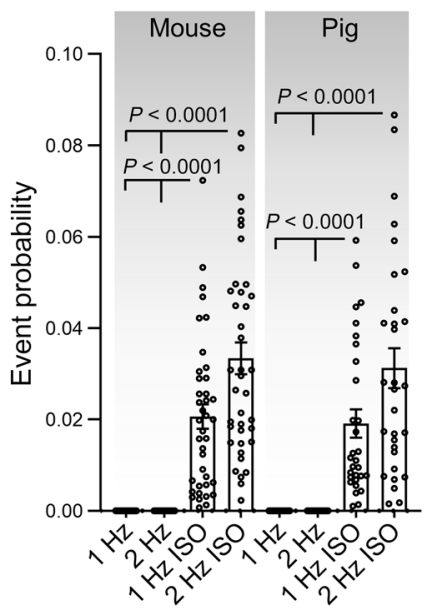

D

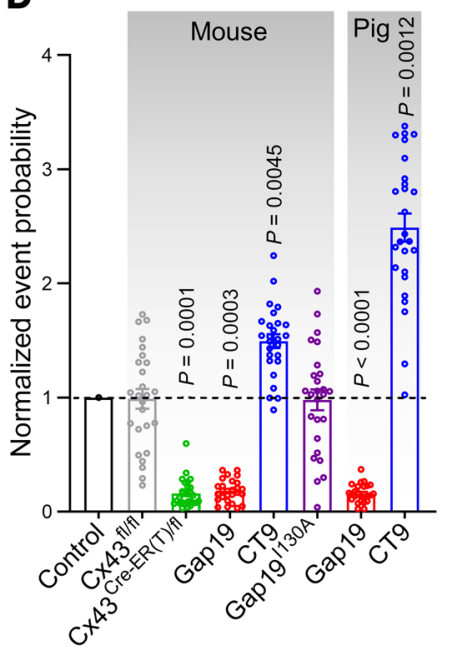

E

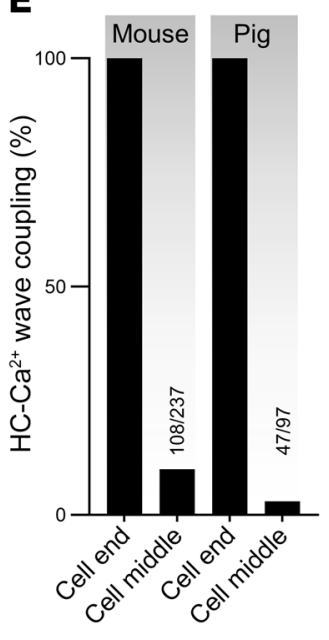

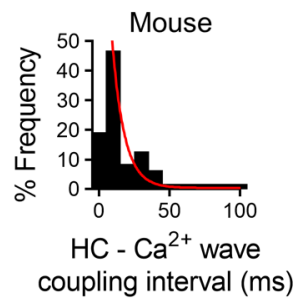

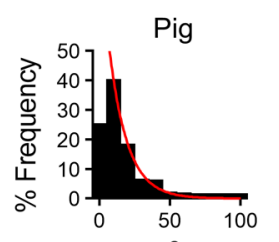

$\mathrm{HC}-\mathrm{Ca}^{2+}$ wave coupling interval (ms)

\section{$\mathbf{F}$}
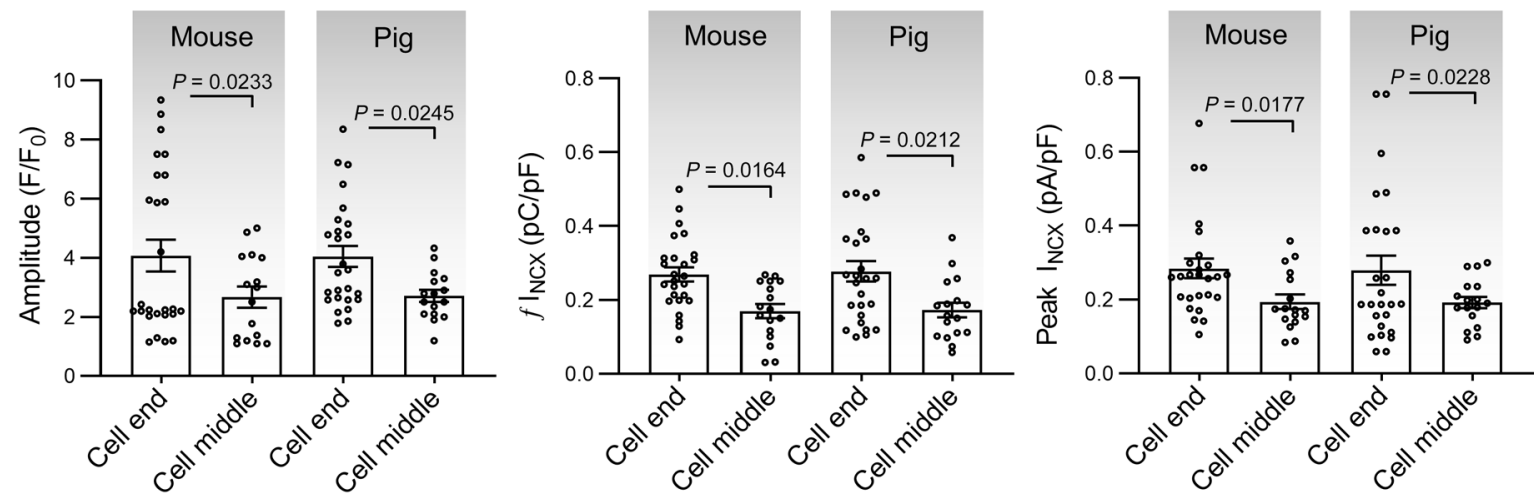

Figure 8. Spontaneous $\mathrm{Cx} 43$ hemichannel openings preceding $\mathrm{Ca}^{2+}$ waves promote arrhythmogenic $\mathrm{Ca}^{2+}$ release and the resulting depolarizing current. (A) Left, unitary currents preceding diastolic Ca ${ }^{2+}$ release. Inset shows detail of unitary current activity. Right, Ca ${ }^{2+}$ images corresponding to time points indicated by dashed lines in left trace. Scale bar: $10 \mu \mathrm{m}$. (B) Transition histograms showing approximately $220 \mathrm{pS}$ unitary conductance of preceding unitary activity $\left(N / n_{\text {mouse }}=23 / 75, N / n_{\text {pig }}=10 / 30\right)$. (C) Summary dot plot illustrating increasing unitary current event probability with increasing pacing frequency and with isoproterenol (nested 1-way ANOVA; $N / n_{\text {mouse }}=23 / 75, N / n_{\text {pig }}=10 / 30$ ). (D) Summary data showing the effect of different interventions at $2 \mathrm{~Hz}+\mathrm{ISO}\left(\mathrm{N} / \mathrm{n}_{\text {mouse }}=5-11 / 15-24\right.$ per condition, $N / n_{\text {pig }}=5 / 15$ per condition). $P$ values indicate significance compared with control (nested 1-way ANOVA). (E) Fraction and coupling interval of $\mathrm{C} x 43 \mathrm{HC}$-associated $\mathrm{Ca}^{2+}$ release $\left(N / n_{\text {mouse }}=23 / 75, N / n_{\text {pig }}=10 / 30\right)$. Left graph indicates that $\mathrm{HC}-\mathrm{Ca} \mathrm{a}^{2+}$ release coupling to $\mathrm{Ca}^{2+}$ waves occurs at the cell end. Numbers show absolute counts. Right histogram indicates time from hemichannel opening to $\mathrm{Ca}^{2+}$ release. (F) Dot plots summarizing properties of diastolic Ca ${ }^{2+}$ release and resulting NCX currents categorized by origin (nested $t$ test; $N / n_{\text {mouse }}=23 / 75, N / n_{\text {pig }}=$ 10/30). HC, hemichannel. 
A

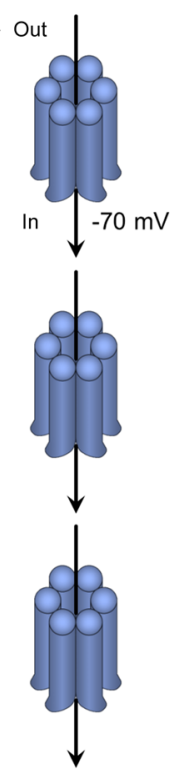

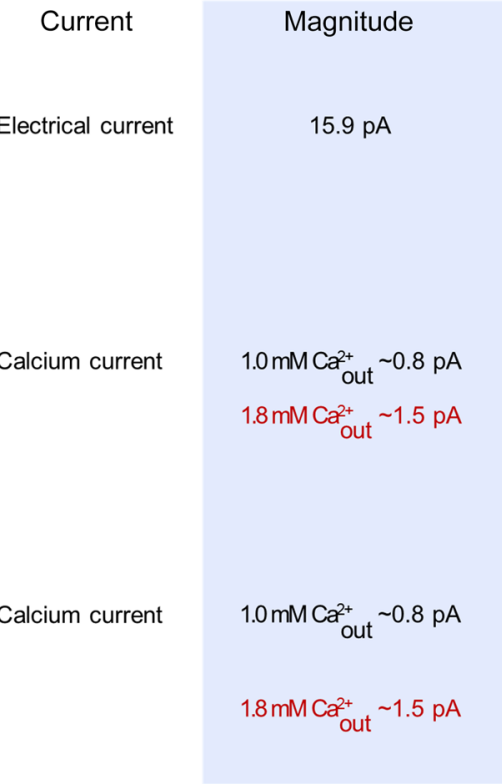

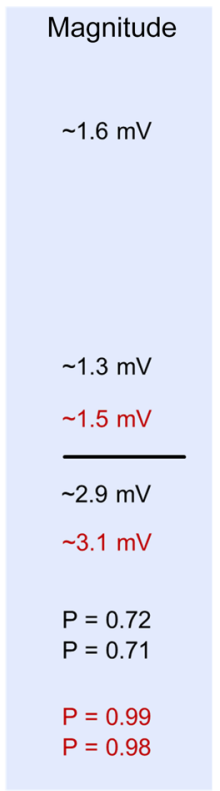

Figure 9. Modeling-based estimations of electrical and $\mathrm{Ca}^{2+}$ consequences of single hemichannel opening. (A) Schematic overview of electrical and $\mathrm{Ca}^{2+}$ consequences of single hemichannel opening. The $15.9 \mathrm{pA}$ electrical current is a measured value; the $0.8 \mathrm{pA}$ and $1.5 \mathrm{pA} \mathrm{Ca}^{2+}$ currents are calculated estimates using 1.0 and $1.8 \mathrm{mM}$ of extracellular $\mathrm{Ca}^{2+}$, respectively. Panels $B$ to $E$ further explore the impact of a range of hemichannel $\mathrm{Ca}^{2+}$ currents. Note that the values given are only valid at $-70 \mathrm{mV}$ membrane potential and $37^{\circ} \mathrm{C}$. Further modeling details can be found in the supplemental material. (B) Peak elevation of subsarcolemmal $\left[\mathrm{Ca}^{2+}\right]_{i}$ as a function of single hemichannel $\mathrm{Ca}^{2+}$ current $\left(I_{\text {са, } \mathrm{HC}}\right)$. (C) Membrane depolarization due to $\mathrm{Ca}^{2+}$ entry is associated with NCX activation. Black and red points indicate hemichannel $\mathrm{Ca}^{2+}$ current estimates, which are close to or in the plateau phase of the curve. (D) Probability of activation of RyR superclusters as a function of single hemichannel $\mathrm{Ca}^{2+}$ current. (E) Probability of $\mathrm{Ca}^{2+}$ wave propagation as a function of single hemichannel $\mathrm{Ca}^{2+}$ current.

D
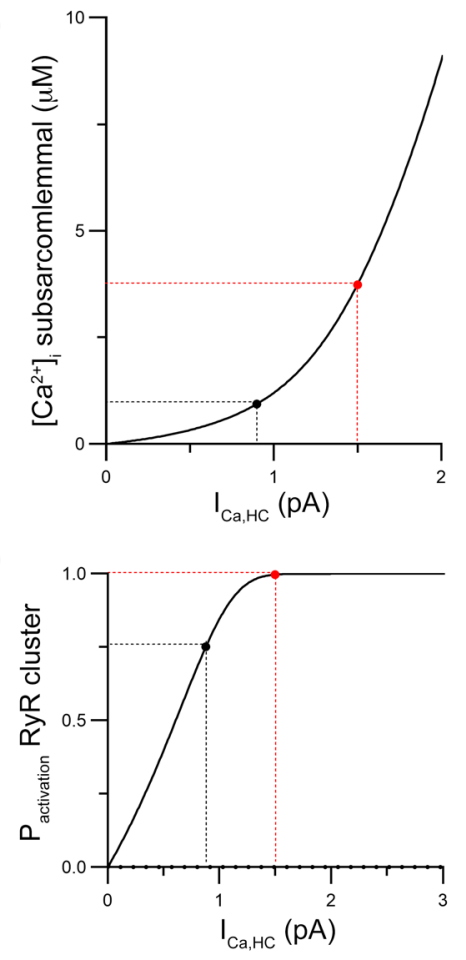

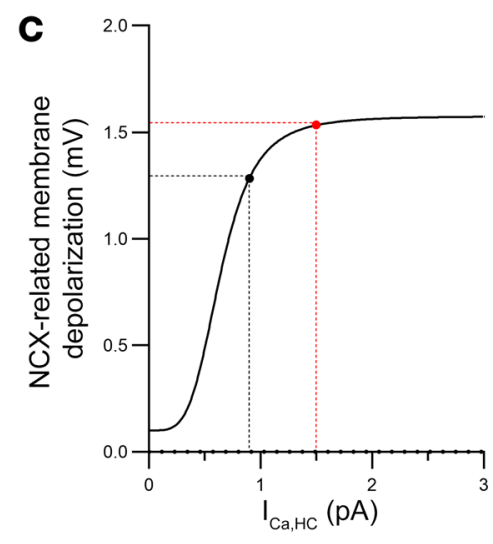

E

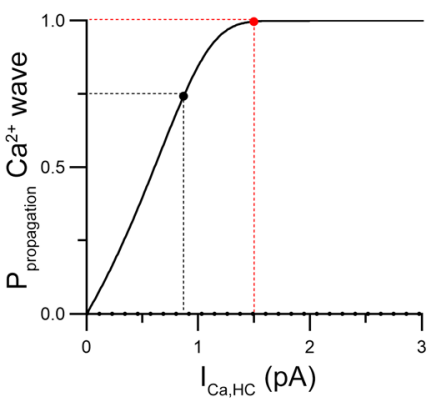

Similar to mouse cardiomyocytes, we observed unitary current activity with approximately 220 pS unitary conductance preceding and during spontaneous diastolic $\mathrm{Ca}^{2+}$ release after adrenergic stimulation (Figure 12, A-C), which was inhibited by Gap19. Additionally, we found that Gap19 significantly decreased spontaneous $\mathrm{Ca}^{2+}$ release and resulting NCX currents in cardiomyocytes from nonfailing and failing hearts (Figure 12, A, D, and E). Current clamp recording revealed significantly increased frequency and amplitude of DADs and triggered action potentials in failing hearts compared with nonfailing hearts, and these events (induced by fast pacing and ISO stimulation) were significantly reduced by TAT-Gap19 (Figure 12, F and G). Finally, monophasic action potential recordings on arterially perfused tissue wedges from failing human hearts showed the highest occurrence of DADs and triggered action potentials after $2 \mathrm{~Hz}$ and ISO stimulation compared with nonfailing wedges, which was significantly reduced by TAT-Gap19 (Figure 12, H and I). Neither in nonfailing nor in failing hearts did we find gross effects of Gap19 on cardiomyocyte $\mathrm{Ca}^{2+}$ transients or SR Ca${ }^{2+}$ content (Supplemental Figure 14A). Similar to mouse cardiomyocytes, we found that $\mathrm{Cx} 43$ hemichannel opening was related to diastolic $\mathrm{Ca}^{2+}$ release originating from the cell end in nonfailing cardiomyocytes; most interestingly, in myocytes from failing hearts, hemichannel opening occurred more frequently in relation to $\mathrm{Ca}^{2+}$ release originating from the cell 
A
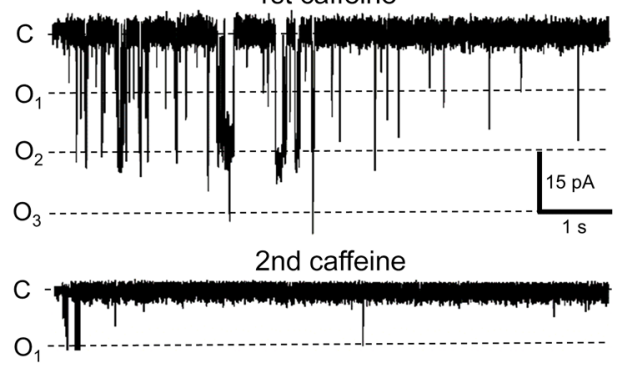

D

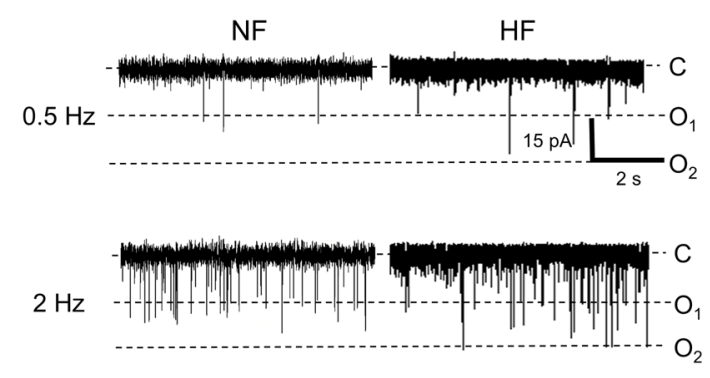

$\mathbf{F}$

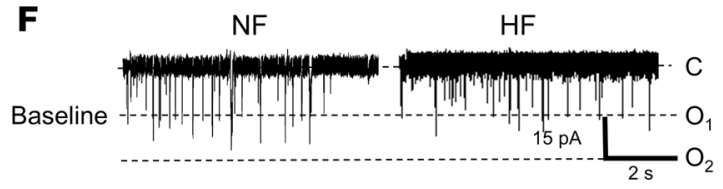

ISO

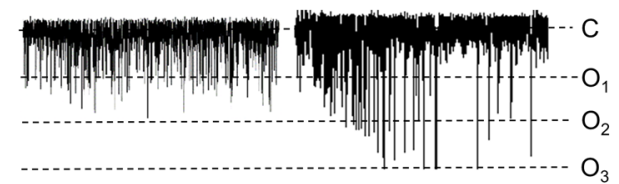

B

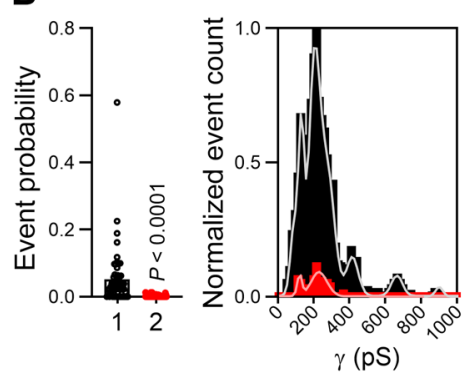

C

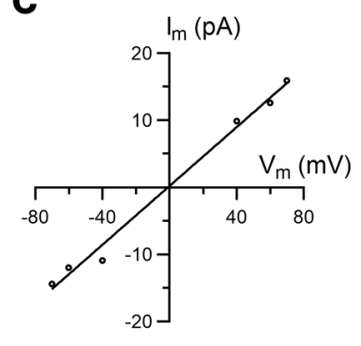

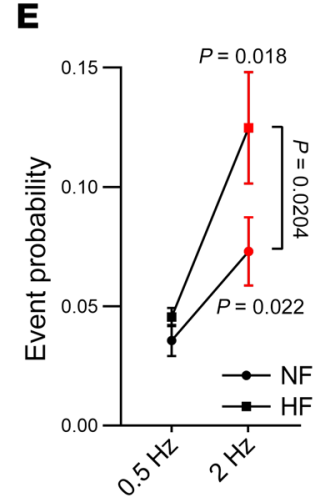

G

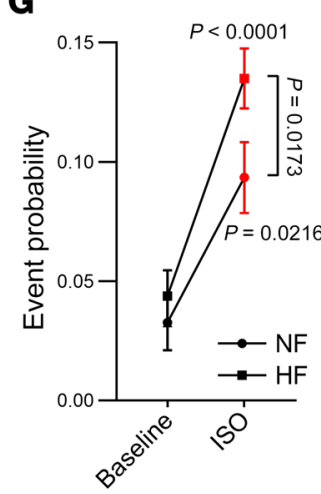

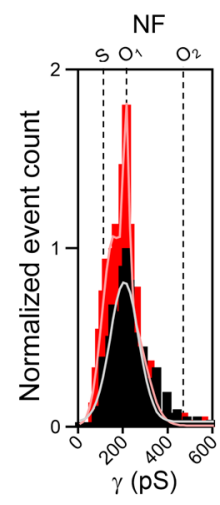

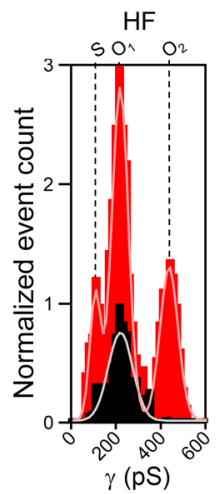

NF
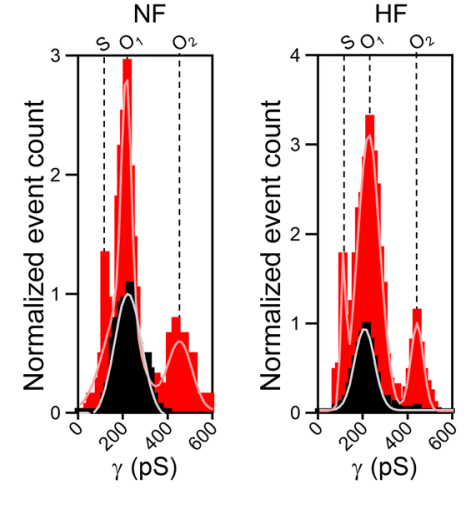

H

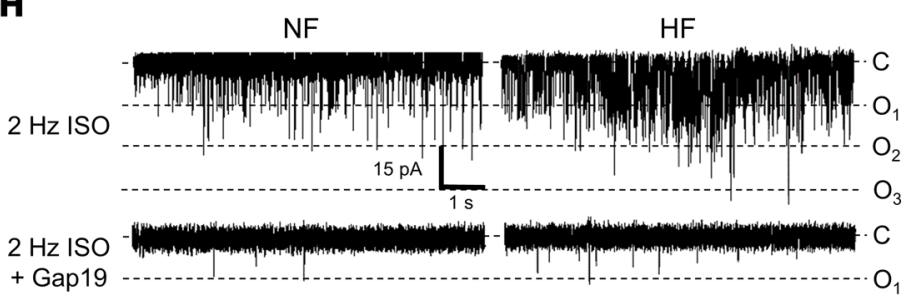

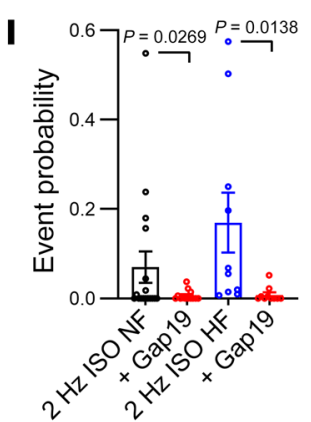

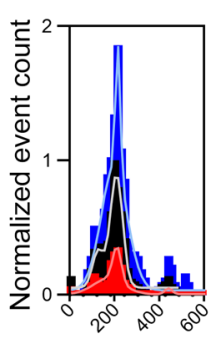

$\gamma(\mathrm{pS})$

Figure 10. Identification and regulation of $\mathbf{C x} 43$ hemichannels in nonfailing and failing human ventricular cardiomyocytes. (A) Unitary current example traces during first and second caffeine applications, NCX current subtracted. Recorded in nonfailing left ventricular human cardiomyocyte. (B) Summary dot plot and transition histogram indicating significantly reduced unitary current event probability during the second caffeine pulse (red) compared with the first (black) (nested $t$ test; $N / n_{\mathrm{NF}}=20 / 64$ ). (C) IV plots depicting linear current-voltage relationship with slope conductance approximately $220 \mathrm{pS}$ and $\mathrm{E}_{\text {rev }} \approx 0 \mathrm{mV}$ $\left(N_{\mathrm{NF}} / n_{\mathrm{NF}}=4 / 14\right)$. (D) Ca $\mathrm{a}^{2+}$ release-induced unitary current example traces following 0.5 and $2 \mathrm{~Hz}$ pacing in nonfailing and failing human cardiac myocytes (NCX currents subtracted). (E) Summary graph and transition histograms indicate significant increase in unitary current event probability with increasing pacing frequency (nested $t$ test; $N / n_{\mathrm{NF}}=5 / 25, N / n_{\mathrm{HF}}=5 / 25$ ). This effect is significantly stronger at $2 \mathrm{~Hz}$ in failing compared with nonfailing cardiomyocytes (nested $t$ test). (F) $\mathrm{Ca}^{2+}$ release-induced unitary current example traces in the absence and presence of isoproterenol $(10 \mathrm{nmol} / \mathrm{L})$ in nonfailing and failing human cardiac myocytes (NCX currents subtracted). (C) Summary graph and transition histograms indicate significant increase in unitary current event probability with isoproterenol compared with baseline (nested $t$ test; $N / n_{\mathrm{NF}}=5 / 13, N / n_{\mathrm{HF}}=5 / 14$ ). The effect was significantly stronger with ISO in failing compared with nonfailing cardiomyocytes (nested $t$ test). ( $\mathbf{H}) \mathrm{Ca}^{2+}$ release-induced unitary current example traces during fast pacing and adrenergic stimulation in nonfailing and failing human cardiomyocytes. Including Gap19 in the pipette solution abolished unitary current activity (NCX currents subtracted). (I) Summary dot plot and transition histogram illustrating increased event probability in failing compared with nonfailing myocytes. Gap19 significantly reduced event probability in nonfailing and failing cardiomyocytes (nested 1-way ANOVA; $N / n_{\mathrm{NF}}=4 / 15, N / n_{\mathrm{HF}}=5 / 15$ ). 
A

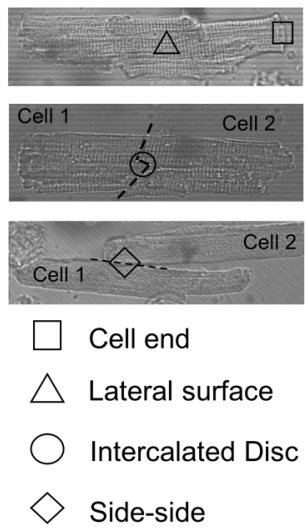

C

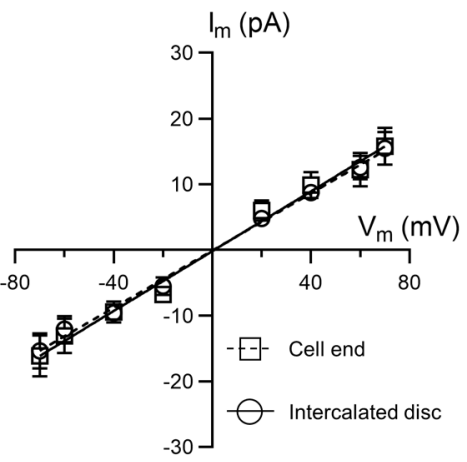

B
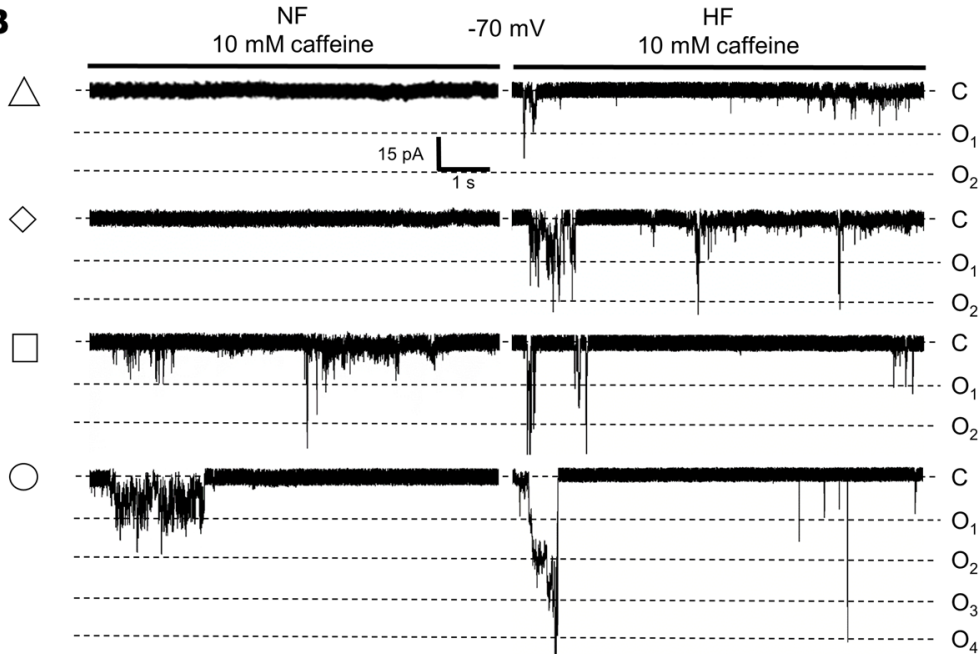

$\mathrm{HF}$

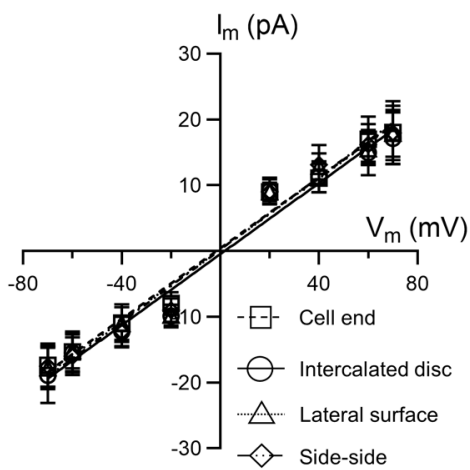

D

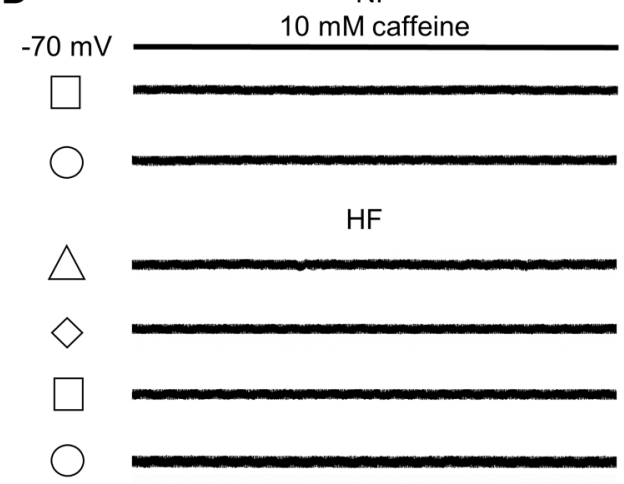

E

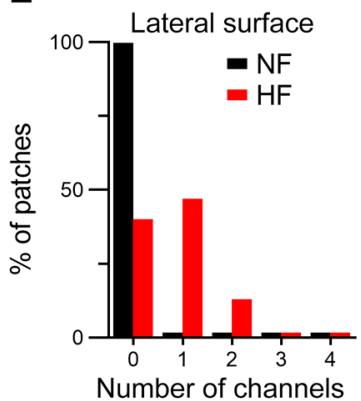

Side-side

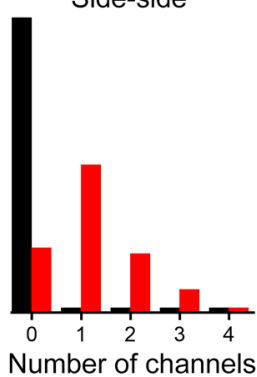

Cell end

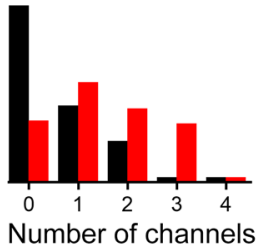

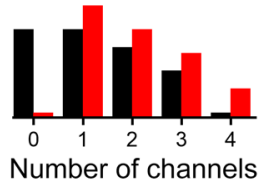

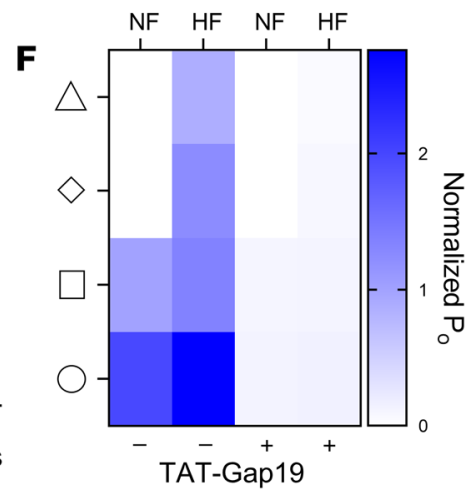

Figure 11. Microdomain-specific activation of Cx43 hemichannels in nonfailing and failing human cardiomyocytes. (A) Transmitted light images of single cardiomyocyte (top) and cardiomyocyte cell pairs (middle, bottom). Triangle, square, circle, and diamond symbols indicate cell-attached macropatch positions at the lateral membrane and cell end of single cardiomyocytes and intercalated disc and side-side of cardiomyocyte cell pairs, respectively. (B) Example traces showing single channel currents recorded at the different macropatch recording positions. Traces recorded in nonfailing and failing human cardiomyocytes during caffeine superfusion (10 mM, 8 seconds) at $-70 \mathrm{mV}$. (C) IV plots depicting linear current-voltage relationship with slope conductance of approximately $220 \mathrm{pS}$ and $\mathrm{E}_{\mathrm{rev}} \approx 0 \mathrm{mV}\left(\mathrm{N} / \mathrm{n}_{\mathrm{NE}}=3 / 10-15\right.$ per recording position, $N / n_{\mathrm{HF}}=3 / 10-15$ per recording position). (D) Example traces showing single channel currents recorded at the different macropatch recording positions following TAT-Gap19 superfusion. (E) Summary histograms depicting number of channels per patch for the different macropatch recording positions $\left(\mathrm{N} / \mathrm{n}_{\mathrm{NF}}=3 / 10-15\right.$ per recording position, $N / n_{\mathrm{HF}}=3 / 10-15$ per recording position). Black and red bars indicate recordings in nonfailing and failing human cardiomyocytes, respectively. (F) Heatmap summarizing single channel open probability at different macropatch recording positions with and without TAT-Gap19 in nonfailing and failing human single cardiomyocytes and cardiomyocyte cell pairs $\left(\mathrm{N} / \mathrm{n}_{\mathrm{NF}}=3 / 10-15\right.$ per recording position, $\mathrm{N} / \mathrm{n}_{\mathrm{HF}}=3 / 10-15$ per recording position). 
A

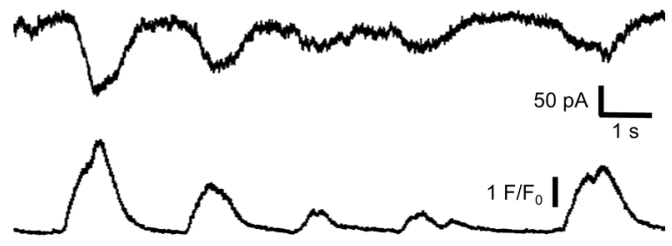

B
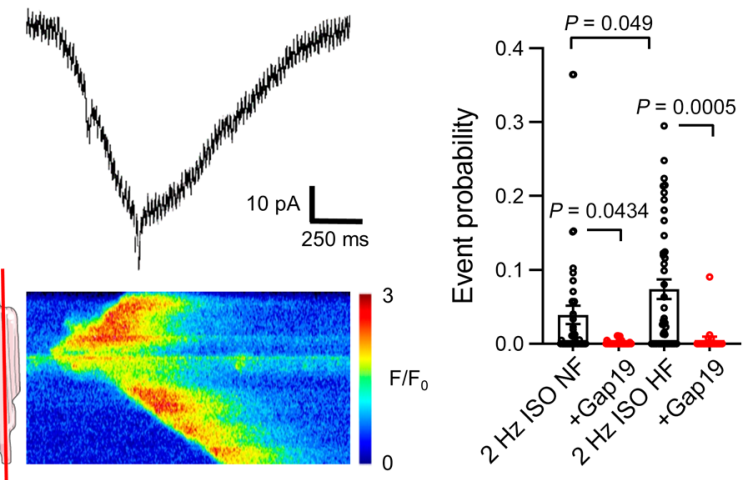

D
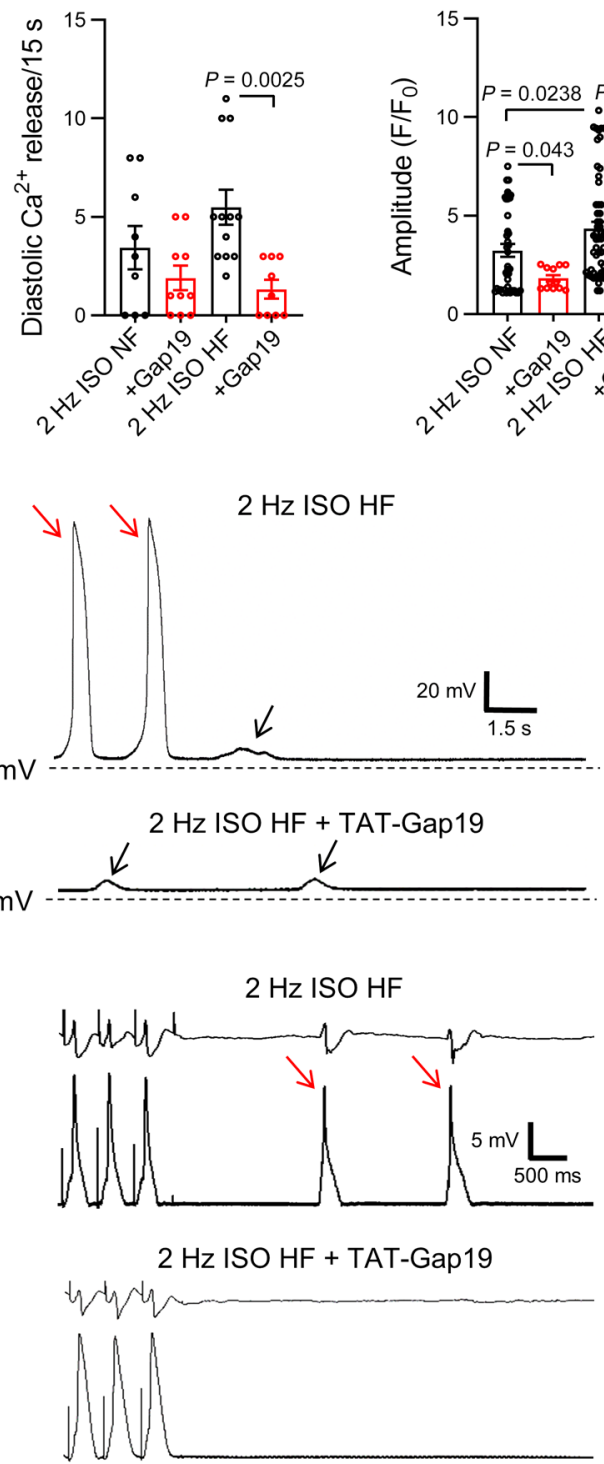

$2 \mathrm{~Hz}$ ISO HF + Gap19

\section{C}
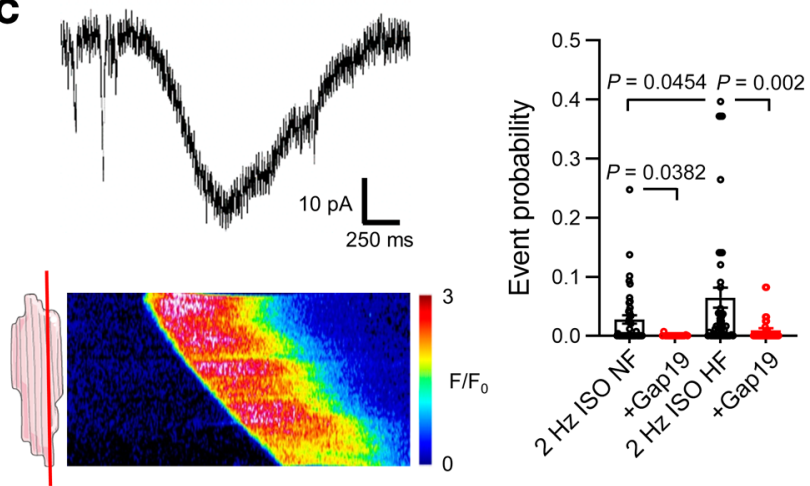

$\mathbf{E}$
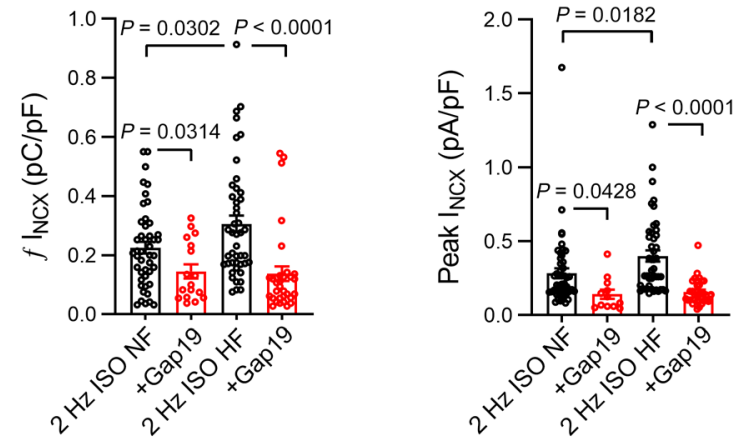

F

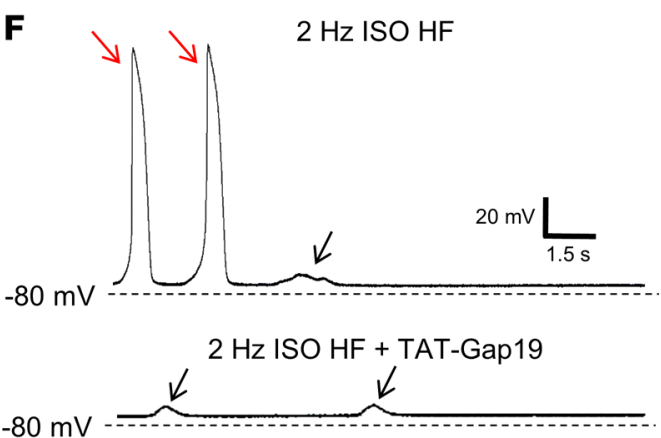

H

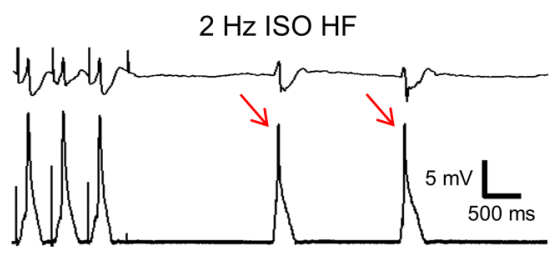

$2 \mathrm{~Hz}$ ISO HF + TAT-Gap19
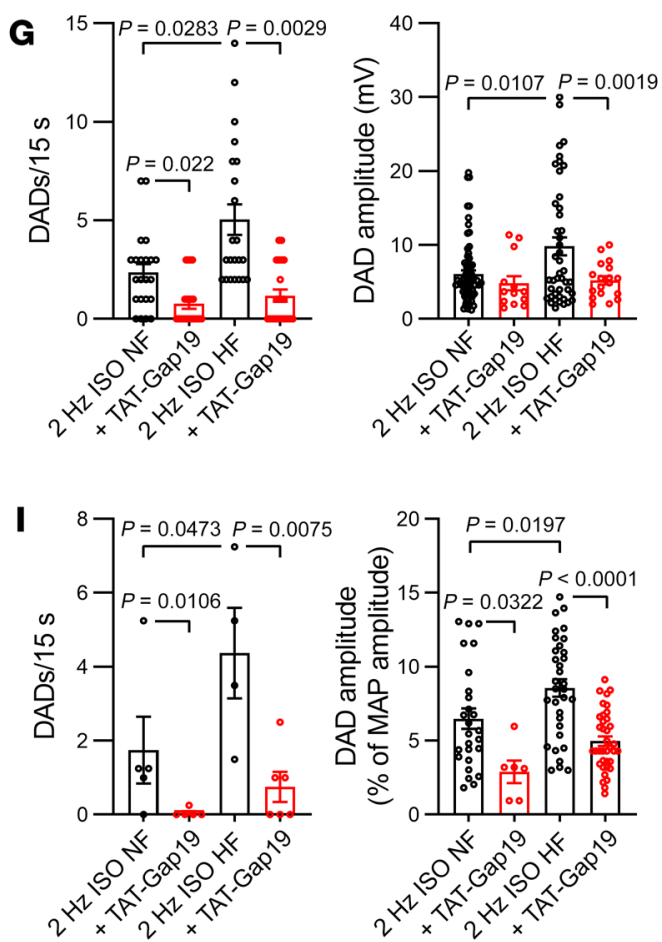
Figure 12. Contribution of $\mathrm{Cx} 43$ hemichannels to spontaneous $\mathrm{Ca}^{2+}$ release and afterdepolarizations in human heart failure. (A) Example traces of spontaneous diastolic $\mathrm{Ca}^{2+}$ release and resulting NCX currents recorded in failing human left ventricular cardiomyocytes following fast pacing and adrenergic stimulation with or without Gap19. (B and C) unitary current detail and associated confocal line scan images during and preceding spontaneous $\mathrm{Ca}^{2+}$ release, respectively. Red line on illustrations indicates scan line position. Dot plots show significant increase in unitary current event probability in failing compared with nonfailing cardiomyocytes. Gap19 abolished unitary current activities (nested 1-way ANOVA; $N / n_{\mathrm{NF}}=$ $\left.5 / 13, N / n_{\mathrm{HF}}=5 / 14\right)$. ( $\mathbf{D}$ and $\mathbf{E}$ ) Summary dot plots illustrating increased frequency and significantly increased amplitude of spontaneous $\mathrm{Ca}^{2+}$ release and associated NCX currents in human heart failure. Gap19 abolished $\mathrm{Ca}^{2+}$ release and NCX currents, especially in heart failure (nested 1-way ANOVA; $\left.N / n_{\mathrm{NF}}=5 / 13, N / n_{\mathrm{HF}}=5 / 14\right)$. (F) Example traces of delayed afterdepolarizations and triggered action potentials recorded in a failing human left ventricular cardiomyocytes at baseline and after TAT-Gap19 superfusion. Black arrows indicate DADs, red arrows indicate triggered action potentials. (C) Summary dot plots showing significantly increased frequency and amplitude of DADs and triggered action potential frequency in failing compared with nonfailing cardiac myocytes. TAT-Gap19 abolished DADs and triggered action potentials, especially in human heart failure (nested 1-way ANOVA; $N / n_{\mathrm{NF}}=6 / 22, N / n_{\mathrm{HF}}=5 / 22$ ). (H) Example ECC (top traces) and monophasic action potential traces (lower traces) recorded during adrenergic stimulation (baseline and after TAT-Gap19) in an arterially perfused left ventricular tissue wedge prepared from failing human heart. Red arrows indicate triggered action potentials. (I) Summary dot plots showing significantly increased frequency and amplitude of DADs and triggered action potential frequency in failing compared with nonfailing tissue wedges. TAT-Gap19 abolished DADs and triggered action potentials, especially in human heart failure (nested 1-way ANOVA; $N / n_{\mathrm{NF}}=5 / 10, N / n_{\mathrm{HF}}=4 / 9$ ).

middle compared with those isolated from nonfailing hearts (Supplemental Figure 14, D and E). Gap19 attenuated both frequency and amplitude of $\mathrm{Cx} 43$ hemichannel-associated $\mathrm{Ca}^{2+}$ release and associated NCX currents (Supplemental Figure 14F).

\section{Discussion}

Our results demonstrated that $\mathrm{Ca}^{2+}$-permeable $\mathrm{Cx} 43$ hemichannels could be activated by $\mathrm{SR} \mathrm{Ca}^{2+}$ release at resting $\mathrm{V}_{\mathrm{m}}$. Microdomain coupling of $\mathrm{Cx} 43$ to distinct dyads at the intercalated disc facilitates this activation and underlies modulation of local $\mathrm{Ca}^{2+}$ release by $\mathrm{Cx} 43$ at these sites. Through both of these mechanisms, $\mathrm{Cx} 43$ hemichannels contribute to triggered activity. $\mathrm{Cx} 43 \mathrm{hemi-}$ channels are more active in human heart failure, especially during adrenergic stimulation, and thereby contribute to electrical instability and arrhythmogenesis in heart failure.

Molecular identity of the large conductance channel during $S R$ $\mathrm{Ca}^{2+}$ release. In 1990, Pott and Mechmann (47) were the first to describe the large conductance channel activated by caffeineinduced or spontaneous $\mathrm{SR} \mathrm{Ca}^{2+}$ release in guinea pig atrial cardiomyocytes. They argued that these channels were possibly related to "cardiac gap junction hemichannels" based on their biophysical profile and pharmacology. Here, we expanded and validated these findings across species, including humans, and demonstrated that Cx43 hemichannels constitute the large conductance channel using genetic and pharmacological tools to complement the biophysical profiling. Over the years, other large conductance channels expressed in cardiomyocytes have been proposed to underlie the spiking unitary current activities superimposed on the NCX current. Sarcolemmal RyRs have been suggested $(48,49)$, but our finding that unitary activity disappears upon SR depletion contradicts the possibility of sarcolemmal RyRs. Transient receptor potential (TRP) channel family members such as PKD2 (TRPP2) or PKD2L2 (TRPP5) as well as Panx1 channels have also been suggested $(25,26)$; however, Panx1 and TRPP2/5 expression were unchanged upon $\mathrm{Cx} 43$ knockdown and ${ }^{10} \mathrm{Panx} 1$ did not influence unitary activity. Instead, the biophysical profile described here, combined with Gap19 hemichannel inhibition and genetic interventions, strongly point to $\mathrm{Cx} 43$ hemichannels as the responsible large conductance channel.

Importantly, this is the first study, to our knowledge, demonstrating that $\mathrm{Cx} 43$ hemichannels may be activated by SR $\mathrm{Ca}^{2+}$ release in cardiomyocyte cell pairs, where gap junctions are present $(50,51)$, indicating that functional $\mathrm{Cx} 43$ hemichannels are present in ventricular myocardium and are not a byproduct of cell dissociation.

$\mathrm{C} x 43$ hemichannels and microdomain $\mathrm{Ca}^{2+}$ signaling. $\mathrm{Cx} 43$ hemichannel activity was modulated by stimulation frequency and ISO. High frequency and beta-adrenergic stimulation enhance microdomain $\mathrm{Ca}^{2+}$ signaling at the dyad $(52,53)$. Dyads are composed of junctional SR that is juxtaposed to L-type $\mathrm{Ca}^{2+}$ channels in T-tubules; electron microscopy-based evidence has indicated that junctional SR is also present at the intercalated disc $(54,55)$. Here, we provide structural and functional support for the existence of a $\mathrm{Cx} 43$ hemichannel-SR $\mathrm{Ca}^{2+}$ release signaling microdomain consisting of large dyadic RyR2 superclusters at the perinexus in healthy myocardium. $\mathrm{Cx} 43$ hemichannel opening had no effect on global cellular $\mathrm{Ca}^{2+}$ dynamics or $\mathrm{SR}^{\mathrm{Ca}^{2+}}$ content, indicating that hemichannels do not contribute to canonical excitation-contraction coupling. However, we showed that $\mathrm{Cx} 43$ hemichannels affected cardiomyocyte electrical excitability by (a) providing direct depolarizing current and (b) $\mathrm{Ca}^{2+}$ entry, increasing NCX current and consequent DAD amplitudes and triggered action potentials. A third level of impact relates to the finding that $\mathrm{Ca}^{2+}$-permeable hemichannels facilitated and increased diastolic $\mathrm{SR} \mathrm{Ca}^{2+}$ release, in particular at the intercalated disc. Numerical simulations corroborated this and indicated that single hemichannel $\mathrm{Ca}^{2+}$ entry into dyads at the intercalated disc highly promoted $\mathrm{Ca}^{2+}$-induced $\mathrm{Ca}^{2+}$ release from neighboring RyR superclusters followed by propagating $\mathrm{Ca}^{2+}$ waves resulting in DADs. Together, these observations may explain the previously observed impact of $\mathrm{Cx} 43$ hemichannel inhibition on adrenergic arrhythmias in muscular dystrophy $(15,16,56)$, arrhythmogenic right ventricular cardiomyopathy (ARVC) (18), and myosin light chain 4 mutationassociated atrial fibrillation (17).

Cx43 hemichannels as an arrhythmogenic mechanism in human heart failure. In human heart failure, we observed a significant increase in hemichannel activity during SR $\mathrm{Ca}^{2+}$ release and adrenergic stimulation. $\mathrm{Cx} 43$ hemichannel inhibition significantly reduced adrenergically mediated diastolic $\mathrm{SR} \mathrm{Ca}^{2+}$ release and associated DADs and triggered action potentials at the cellular level as well as in arterially perfused tissue wedges from nonfailing and failing human left ventricle. As in mouse experiments, Cx43 hemichannel inhibition had no gross effects on cellular $\mathrm{Ca}^{2+}$ signaling or $\mathrm{SR} \mathrm{Ca}^{2+}$ content in nonfailing and failing cardiomyocytes. Yet, hemichannels enhanced diastolic SR $\mathrm{Ca}^{2+}$ release, 
presumably through microdomain $\mathrm{Ca}^{2+}$ signaling. Interestingly, $\mathrm{Cx} 43$ events and the coupling to $\mathrm{Ca}^{2+}$ were observed at the cell ends and in the middle, reflecting a shift in Cx43 location and RyR activity (54). Indeed, macropatch recording showed that in heart failure - hemichannel activity may redistribute from the cell ends to the lateral membranes of single cardiomyocytes and cardiomyocyte cell pairs. Taken together, these data provide evidence that $\mathrm{Cx} 43$ hemichannels function as a ventricular arrhythmogenic mechanism that can be targeted by Gap19 without negative impact on inotropy.

A limitation of this study is that numerical simulations were based on (a) several assumptions and only give an order of magnitude of the effect, and (b) the functional and structural data were obtained in control cardiomyocytes, not in diseased cells. This may be the reason why the calculated probability of single hemichannel opening leading to $\mathrm{Ca}^{2+}$ waves was somewhat higher than observed in the experiments (0.71 vs. 0.5). Second, in the human heart experiments, control nonfailing cardiomyocytes were obtained from hearts that were not suitable for transplantation for various reasons (e.g., age, comorbidity), implying that these controls may differ from what is considered healthy.

\section{Methods}

We utilized commercially available C57/BL6 mice (obtained from Invigo) and healthy control pigs (obtained from the Zootechnical Center). Additionally, we used an established inducible $\mathrm{Cx} 43$ knockdown mouse model (Cx43 $3^{\mathrm{Cre}-\mathrm{ER}(\mathrm{T}) / \mathrm{fl}}$ mice, refs. 57-63). Daily i.p. injection of $3 \mathrm{mg}$ 4-hydroxytamoxifen (4-OHT; Sigma-Aldrich) dissolved in sunflower oil for 5 consecutive days induced Cre-ER(T) activity, which progressively deleted the floxed $\mathrm{Cx} 43$ allele in adult $\mathrm{Cx} 43^{\mathrm{Cre}-\mathrm{ER}(\mathrm{T}) / \mathrm{fl}}$ mice. Experimental work was performed on day 11 after the first injection; 4-OHT-treated $\mathrm{Cx} 43^{\mathrm{t} / \mathrm{l} l}$ mice were used as controls. Animals were housed in a licensed facility and handled in accordance with European Directive 2010/63/EU. For human studies, we used rejected donor hearts as well as explanted failing hearts. Patient characteristics are summarized in Supplemental Table 8.

Procedures used for cardiomyocyte isolation, cellular electrophysiology, and simultaneous $\mathrm{Ca}^{2+}$ imaging in single ventricular cardiomyocytes, DNA extraction, PCR, Western blots, confocal and electron and single-molecule localization microscopy, cell culture, siRNA transfection, macropatch and super-resolution patch clamp, monophasic action potential recording in ventricular tissue wedges, and numerical simulations follow previously published protocols and are detailed in Supplemental Methods.

Synthetic peptides used in this study to interfere with $\mathrm{Cx} / \mathrm{Panx}$ channel function were all obtained from Pepnome. Gap19, Gap191130A, and CT9 were added to the pipette solution at a concentration of 100 $\mu \mathrm{mol} / \mathrm{L}$. TAT-Gap19, TAT-Gap19 ${ }^{1130 \mathrm{~A}}$, and TAT-CT9 were added to the bath solution at $80 \mu \mathrm{mol} / \mathrm{L} .{ }^{10} \mathrm{Panx} 1$, a blocker of Panx1 channels, was preincubated at $200 \mu \mathrm{mol} / \mathrm{L}$ for 30 minutes and present in the extracellular solution during whole cell recording. For macropatch experiments, ${ }^{10} \mathrm{Panx} 1$ was included in the pipette solution at $200 \mu \mathrm{mol} / \mathrm{L}$. The identity of the peptides was confirmed by mass spectrometry and purity was $90 \%$ or greater.

Statistics. Data are expressed as mean \pm SEM unless otherwise indicated, with $n$ denoting the number of cells and $N$ denoting the number of independent experiments. After outlier analysis (mean \pm
$3 \times \mathrm{SD}$ ), normality of distribution was tested, and the appropriate statistical test was determined for comparative statistics. Data were compared using a nested design, taking into account $n$ cells and $N$ animals or human hearts. A 2-tailed $P$ value less than 0.05 was considered as indicating statistical significance. In the graphs, the actual $P$ values are provided. Statistical analysis and graphical data representation were done with GraphPad Prism (v.9).

Study approval. Experimental methods were approved by the local ethics committees for animal research at Ghent University, KU Leuven, and New York University School of Medicine (NYU IACUC).

For human studies, experimental procedures were approved by the Ethical Committee of the University Hospital of Leuven with permit number S58824; because this was residual tissue, no informed consent was necessary.

\section{Author contributions}

MDS designed, conducted, and analyzed most of the experiments and wrote and revised the manuscript with input from all the authors. AL contributed to experimental design. TN designed the software for analysis of membrane currents and numerical simulations. NW contributed to the experimental design. ED contributed to cardiomyocyte cell isolation and cellular electrophysiology. $\mathrm{MPH}$ contributed to the design and analysis of super-resolution and electron microscopy experiments. XL performed SICM-guided patch clamp experiments. MA contributed to cardiomyocyte cell isolation, cellular electrophysiology, and monophasic action potential recording. TV performed and analyzed all experiments with tetracycline-inducible RyR2-overexpressing cells. KW contributed to single-channel analysis. ER guided experimental design and provided computational tools for processing and analysis of super-resolution fluorescence microscopy. GB guided experiments in tetracycline-inducible RyR2-overexpressing cells. RS provided inducible Cx43 knockdown mice. AVP guided design of analysis software and numerical simulations. MD, KRS, and LL conceived, initiated, and supervised the project; gave scientific input; and revised the manuscript.

\section{Acknowledgments}

We sincerely thank Ellen Cocquyt, Diego De Baere, Vicky Pauwelyn, Annemie Biesemans, Roxane Menten, and Mingliang Zhang for superb technical support. We would also like to thank the heart failure unit, the transplant surgical team, and the transplant coordinating team of UZ Leuven for help in providing the human explant hearts. This work was supported by the Fund for Scientific Research Flanders (project grants to LL, KRS, and GB; a postdoctoral fellowship to ED; and PhD fellowships to MDS and MA); Ghent University (a postdoctoral fellowship to KW and PhD fellowships to AL and TN); the Interuniversity Attraction Poles P7/10 to KRS and LL; NIH (project grants to ER and MD); the Fondation Leducq (transatlantic network award to MD); and a grant from the Ministry of Science and Higher Education of the Russian Federation, agreement 075-15-2020-800, to AVP.

Address correspondence to: Luc Leybaert, Ghent University, Campus UZ Gent Block B, $3^{\text {rd }}$ Floor, Corneel Heymanslaan 10, B-9000 Ghent, Belgium. Phone: 32.9.3323366; Email Luc. Leybaert@UGent.be. 
1. Giovannone S, et al. Channeling diversity: gap junction expression in the heart. Heart Rhythm. 2012;9(7):1159-1162.

2. Rhett JM, et al. The perinexus: sign-post on the path to a new model of cardiac conduction? Trends Cardiovasc Med. 2013;23(6):222-228.

3. Wang $\mathrm{N}$, et al. Connexin mimetic peptides inhibit $\mathrm{Cx} 43$ hemichannel opening triggered by voltage and intracellular Ca2+ elevation. Basic Res Cardiol. 2012;107(6):304.

4. Wang $\mathrm{N}$, et al. Selective inhibition of $\mathrm{Cx} 43$ hemichannels by Gap19 and its impact on myocardial ischemia/reperfusion injury. Basic Res Cardiol. 2013;108(1):309.

5. Bol M, et al. At the cross-point of connexins, calcium, and ATP: blocking hemichannels inhibits vasoconstriction of rat small mesenteric arteries. Cardiovasc Res. 2017;113(2):195-206.

6. Contreras JE, et al. Gating and regulation of connexin 43 (Cx43) hemichannels. Proc Natl Acad Sci U S A. 2003;100(20):11388-11393.

7. Freitas-Andrade M, et al. Targeting MAPK phosphorylation of Connexin 43 provides neuroprotection in stroke. J Exp Med. 2019;216(4):916-935.

8. Gadicherla AK, et al. Mitochondrial Cx43 hemichannels contribute to mitochondrial calcium entry and cell death in the heart. Basic Res Cardiol. 2017;112(3):27.

9. Meunier C, et al. Contribution of astroglial $\mathrm{Cx} 43$ hemichannels to the modulation of glutamatergic currents by $\mathrm{D}$-serine in the mouse prefrontal cortex. J Neurosci. 2017;37(37):9064-9075.

10. Gilbert $\mathrm{G}$, et al. Calcium signaling in cardiomyocyte function. Cold Spring Harb Perspect Biol. 2020;12(3):a035428.

11. Antoons G, et al. Alternative strategies in arrhythmia therapy: evaluation of $\mathrm{Na} / \mathrm{Ca}$ exchange as an anti-arrhythmic target. Pharmacol Ther. 2012;134(1):26-42.

12. Landstrom AP, et al. Calcium signaling and cardiac arrhythmias. Circ Res. 2017;120(12):1969-1993.

13. Sipido KR, et al. $\mathrm{Na} / \mathrm{Ca}$ exchange and cardiac ventricular arrhythmias. Ann N Y Acad Sci. 2007;1099:339-348.

14. Leybaert L, et al. Connexins in cardiovascular and neurovascular health and disease: pharmacological implications. Pharmacol Rev. 2017;69(4):396-478.

15. Lillo MA, et al. S-nitrosylation of connexin 43 hemichannels elicits cardiac stress-induced arrhythmias in Duchenne muscular dystrophy mice. JCI Insight. 2019;4(24):e130091.

16. Patrick Gonzalez J, et al. Selective Connexin 43 inhibition prevents isoproterenol-induced arrhythmias and lethality in muscular dystrophy mice. Sci Rep. 2015;5:13490.

17. Ghazizadeh Z, et al. Metastable atrial state underlies the primary genetic substrate for MYL4 mutation-associated atrial fibrillation. Circulation. 2020;141(4):301-312.

18. Kim J-C, et al. Disruption of $\mathrm{Ca}^{2+}{ }_{\mathrm{i}}$ homeostasis and connexin 43 hemichannel function in the right ventricle precedes overt arrhythmogenic cardiomyopathy in plakophilin-2-deficient mice. Circulation. 2019;140(12):1015-1030.

19. Murayama T, et al. A tryptophan residue in the caffeine-binding site of the ryanodine receptor regulates $\mathrm{Ca}^{2+}$ sensitivity. Commun Biol.
2018;1(1):98.

20. Eisner DA, et al. Calcium and excitationcontraction coupling in the heart. Circ Res. 2017;121(2):181-195.

21. Lissoni A, et al. RyR2 regulates Cx43 hemichannel intracellular $\mathrm{Ca} 2+$-dependent activation in cardiomyocytes. Cardiovasc Res. 2021;117(1):123-136.

22. Trafford AW, et al. Comparison of subsarcolemmal and bulk calcium concentration during spontaneous calcium release in rat ventricular myocytes. J Physiol. 1995;488(3):577-586.

23. Weber CR, et al. $\mathrm{Na}^{+}-\mathrm{Ca}^{2+}$ exchange current and submembrane $\left[\mathrm{Ca}^{2+}\right]$ during the cardiac action potential. Circ Res. 2002;90(2):182-189.

24. De Bock M, et al. Connexin 43 hemichannels contribute to cytoplasmic $\mathrm{Ca}^{2+}$ oscillations by providing a bimodal $\mathrm{Ca}^{2+}$-dependent $\mathrm{Ca}^{2+}$ entry pathway. J Biol Chem. 2012;287(15):12250-1266.

25. Kienitz M-C, et al. Pannexin 1 constitutes the large conductance cation channel of cardiac myocytes. JBiol Chem. 2011;286(1):290-298.

26. Volk T, et al. A polycystin-2-like large conductance cation channel in rat left ventricular myocytes. Cardiovasc Res. 2003;58(1):76-88.

27. Bermudez-Hernandez K, et al. A method for quantifying molecular interactions using stochastic modelling and super-resolution microscopy. Sci Rep. 2017;7(1):14882.

28. Jayasinghe ID, et al. Organization of ryanodine receptors, transverse tubules, and sodiumcalcium exchanger in rat myocytes. Biophys J. 2009;97(10):2664-2673.

29. Shen $X$, et al. 3D dSTORM imaging reveals novel detail of ryanodine receptor localization in rat cardiac myocytes. J Physiol. 2019;597(2):399-418.

30. Macquaide $\mathrm{N}$, et al. Ryanodine receptor cluster fragmentation and redistribution in persistent atrial fibrillation enhance calcium release. Cardiovasc Res. 2015;108(3):387-398.

31. Veeraraghavan R, Gourdie RG. Stochastic optical reconstruction microscopy-based relative localization analysis (STORM-RLA) for quantitative nanoscale assessment of spatial protein organization. Mol Biol Cell. 2016;27(22):3583-3590.

32. Veeraraghavan $\mathrm{R}$, et al. The adhesion function of the sodium channel beta subunit ( $\beta 1$ ) contributes to cardiac action potential propagation. Elife. 2018;7:e37610.

33. Rhett JM, Gourdie RG. The perinexus: a new feature of $\mathrm{Cx} 43$ gap junction organization. Heart Rhythm. 2012;9(4):619-623.

34. Rhett JM, et al. Cx43 associates with Nav1.5 in the cardiomyocyte perinexus. J Membr Biol. 2012;245(7):411-422.

35. Unger VM, et al. Three-dimensional structure of a recombinant gap junction membrane channel. Science. 1999;283(5405):1176-1180.

36. Page E, et al. Freeze-fractured cardiac gap junctions: structural analysis by three methods. Am J Physiol. 1983;244(4):H525-H539.

37. Rhett JM, et al. Connexin 43 connexon to gap junction transition is regulated by zonula occludens-1. Mol Biol Cell. 2011;22(9):1516-1528.

38. Lin X, et al. Subcellular heterogeneity of sodium current properties in adult cardiac ventricular myocytes. Heart Rhythm. 2011;8(12):1923-1930.

39. Jiang D, et al. RyR2 mutations linked to ventricular tachycardia and sudden death reduce the threshold for store-overload-induced $\mathrm{Ca} 2+$ release (SOICR). Proc Natl Acad SciU S A. 2004;101(35):13062-13067.

40. Dibb KM, et al. Regulation of systolic $\left[\mathrm{Ca}^{2+}\right] \mathrm{i}$ and cellular $\mathrm{Ca}^{2+}$ flux balance in rat ventricular myocytes by SR Ca ${ }^{2+}$, L-type $\mathrm{Ca}^{2+}$ current and diastolic $\left[\mathrm{Ca}^{2+}\right]$ i. J Physiol. 2007;585(2):579-592.

41. Sankaranarayanan $\mathrm{R}$, et al. Systolic $\left[\mathrm{Ca}^{2+}\right]$ i regulates diastolic levels in rat ventricular myocytes. JPhysiol. 2017;595(16):5545-5555.

42. Sipido KR, et al. Low efficiency of $\mathrm{Ca} 2+$ entry through the $\mathrm{Na}(+)-\mathrm{Ca} 2+$ exchanger as trigger for $\mathrm{Ca} 2+$ release from the sarcoplasmic reticulum. A comparison between L-type Ca2+ current and reverse-mode $\mathrm{Na}(+)-\mathrm{Ca} 2+$ exchange. Circ Res. 1997;81(6):1034-1044.

43. Trafford AW, et al. Modulation of CICR has no maintained effect on systolic $\mathrm{Ca} 2+$ : simultaneous measurements of sarcoplasmic reticulum and sarcolemmal Ca2+ fluxes in rat ventricular myocytes. J Physiol. 2000;522(2):259-270.

44 . Venetucci LA, et al. Reducing ryanodine receptor open probability as a means to abolish spontaneous $\mathrm{Ca} 2+$ release and increase $\mathrm{Ca} 2+$ transient amplitude in adult ventricular myocytes. Circ Res. 2006;98(10):1299-1305.

45. Cannell MB, Soeller C. Numerical analysis of ryanodine receptor activation by L-type channel activity in the cardiac muscle diad. Biophys $J$. 1997;73(1):112-122.

46. Tao T, et al. Alternans of cardiac calcium cycling in a cluster of ryanodine receptors: a simulation study. Am J Physiol Heart Circ Physiol. 2008;295(2):H598-H609.

47. Pott L, Mechmann S. Large-conductance ion channel measured by whole-cell voltage clamp in single cardiac cells: modulation by beta-adrenergic stimulation and inhibition by octanol. JMembr Biol.1990;117(2):189-199.

48. Kondo RP, et al. Putative ryanodine receptors in the sarcolemma of ventricular myocytes. Pflugers Arch. 2000;440(1):125-131.

49. Zhang YA, et al. Caffeine-activated large-conductance plasma membrane cation channels in cardiac myocytes: characteristics and significance. Am J Physiol Heart Circ Physiol. 2007;293(4):H2448-H2461.

50. Hagen A, et al. Human cardiac gap-junction coupling: effects of antiarrhythmic peptide AAP10. Cardiovasc Res. 2009;83(2):405-415.

51. Li Y, et al. Do calcium waves propagate between cells and synchronize alternating calcium release in rat ventricular myocytes? J Physiol. 2012;590(24):6353-6361.

52. Dries E, et al. Selective modulation of coupled ryanodine receptors during microdomain activation of calcium/calmodulin-dependent kinase II in the dyadic cleft. Circ Res. 2013;113(11):1242-1252.

53. Dries E, et al. Calcium/calmodulin-dependent kinase II and nitric oxide synthase 1-dependent modulation of ryanodine receptors during $\beta$-adrenergic stimulation is restricted to the dyadic cleft. J Physiol. 2016;594(20):5923-5939.

54. Bennett PM, et al. Sarcoplasmic reticulum is an intermediary of mitochondrial and myofibrillar growth at the intercalated disc. JMuscle Res Cell Motil. 2016;37(3):55-69.

55. Leo-Macías A, et al. Ultrastructure of the inter- 


\section{RESEARCH ARTICLE}

cellular space in adult murine ventricle revealed by quantitative tomographic electron microscopy. Cardiovasc Res. 2015;107(4):442-452.

56. Himelman E, et al. Prevention of Connexin 43 remodeling protects against Duchenne muscular dystrophy cardiomyopathy. JClin Invest. 2020;130(4):1713-1727.

57. Boengler K, et al. Mitochondrial connexin 43 impacts on respiratory complex I activity and mitochondrial oxygen consumption. J Cell Mol Med. 2012;16(8):1649-1655.

58. Boengler K, et al. Connexin 43 impacts on mito- chondrial potassium uptake. Front Pharmacol. 2013;4:73.

59. Eckardt D, et al. Functional role of connexin 43 gap junction channels in adult mouse heart assessed by inducible gene deletion. JMol Cell Cardiol. 2004;36(1):101-110.

60. Jansen JA, et al. Reduced heterogeneous expression of Cx43 results in decreased Nav1.5 expression and reduced sodium current that accounts for arrhythmia vulnerability in conditional $\mathrm{Cx} 43$ knockout mice. Heart Rhythm. 2012;9(4):600-607.

61. Jansen JA, et al. Reduced Cx43 expression
The Journal of Clinical Investigation

triggers increased fibrosis due to enhanced fibroblast activity. Circ Arrhythm Electrophysiol. 2012;5(2):380-390.

62. Stein M, et al. A 50\% reduction of excitability but not of intercellular coupling affects conduction velocity restitution and activation delay in the mouse heart. PLoS One. 2011;6(6):e20310.

63. van Rijen HVM, et al. Slow conduction and enhanced anisotropy increase the propensity for ventricular tachyarrhythmias in adult mice with induced deletion of connexin43. Circulation. 2004;109(8):1048-1055. 